\title{
Ideals of independence
}

\author{
Vera Fischer $^{1}$. Diana Carolina Montoya ${ }^{1}(0$ \\ Received: 5 February 2019 / Accepted: 8 March 2019 / Published online: 15 March 2019 \\ (c) The Author(s) 2019
}

\begin{abstract}
We study two ideals which are naturally associated to independent families. The first of them, denoted $\mathcal{J}_{\mathcal{A}}$, is characterized by a diagonalization property which allows along a cofinal sequence (the order type of which of uncountable cofinality) of stages along a finite support iteration to adjoin a maximal independent family. The second ideal, denoted $\operatorname{id}(\mathcal{A})$, originates in Shelah's proof of $\mathfrak{i}<\mathfrak{u}$ in Shelah (Arch Math Log 31(6), 433-443, 1992). We show that for every independent family $\mathcal{A} \operatorname{id}(\mathcal{A}) \subseteq \mathcal{J}_{\mathcal{A}}$ and define a class of maximal independent families, to which we refer as densely maximal, for which the two ideals coincide. Building upon the techniques of Shelah (1992) we characterize Sacks indestructibility for such families in terms of properties of $\operatorname{id}(\mathcal{A})$ and devise a countably closed poset which adjoins a Sacks indestructible densely maximal independent family.
\end{abstract}

Keywords Independent families · Sacks indestructibility · Constellations of cardinal characteristics

Mathematics Subject Classification 03E17 · 03E35

\section{Introduction}

The study of various combinatorial sets of reals, among which maximal almost disjoint families, maximal cofinitary groups and towers, occupy a central place in modern set theory. In this article we study maximal independent families. The concept of independence first appeared in a paper of Fichtenholz and Kantorovic titled Sur les

The authors would like to thank the Austrian Science Fund (FWF) for the generous support through START Grant Y1012-N35.

$凶$ Diana Carolina Montoya

diana.carolina.montoya.amaya@univie.ac.at

Vera Fischer

vera.fischer@univie.ac.at

1 Kurt Gödel Research Center, University of Vienna, Währinger Strasse 25, 1090 Vienna, Austria 
opérations linéaries dans l'espace des fonctions bornées [6] in which they study properties of linear functionals in the space of bounded measurable functions and define the notion of independence for measurable subsets of the unit interval. The contemporary terminology is as follows:

Definition 1 (Notation) Let $\mathcal{A}$ be a family of infinite subsets of $\omega$ :

- We call $\operatorname{FF}(\mathcal{A})$ the family of finite partial functions from $\mathcal{A}$ to 2 .

- Given $h \in \operatorname{FF}(\mathcal{A}), \mathcal{A}^{h}=\bigcap\left\{A^{h(A)}: A \in \mathcal{A} \cap \operatorname{dom}(h)\right\}$, where $A^{h(A)}=A$ if $h(A)=0$ and $A^{h(A)}=\omega \backslash A$ otherwise.

- We refer to $\left\{\mathcal{A}^{h}: h \in \operatorname{FF}(\mathcal{A})\right\}$ is the family of Boolean combinations of $\mathcal{A}$.

Definition 2 A family $\mathcal{A} \subseteq[\omega]^{\omega}$ is called independent if for for every $h \in \operatorname{FF}(\mathcal{A})$, the set $\mathcal{A}^{h}$ is infinite. An independent family $\mathcal{A}$ is said to be maximal independent if it is not properly contained in another independent family.

Fichtenholz and Kantorovic also prove in [6] (Lemma II) that there is an independent family of size $\mathfrak{c}$ (see also [10] to find many different examples of such objects). This result was generalized by Hausdorff in [13], who showed that for every infinite cardinal $\kappa$, there is an independent family of size $2^{\kappa}$. Clearly, Zorn's lemma guarantees then the existence of maximal independent families of size $2^{\kappa}$ for all $\kappa$. Particular interest has been given to the independence number $\mathfrak{i}$, which is defined as the minimum size of a maximal independent family. ${ }^{1}$ Well-known lower bounds of the independence number are $\mathfrak{r}, \mathfrak{d}$, the reaping and the dominating numbers respectively (see $[2,12]$ ). However, there are not known upper bounds, except for the trivial one $\mathfrak{c}$. The value of the independence number has been calculated in many well-known forcing extensions (see [2] for details), for instance, in generic extensions in which we cofinally add $\lambda$-many Cohen reals, we have that $i \geq \lambda$ because Cohen reals split the ground model, forcing the value of $\mathfrak{r}$ to be $\geq \lambda$. As consequence, in the Cohen, Hechler, Random (with finite support) and Mathias extensions the value of $i$ is $\mathfrak{c}$. On the other hand, in classical models obtained as countable support iterations we have the following: In the Miller and the Laver extensions $\mathfrak{i}=\mathfrak{c}$, in the first case this is a consequence of the fact that Miller functions are unbounded, so $\mathfrak{d}=\mathfrak{c}$, and in the second we know that Laver reals are dominating, so $\mathfrak{b}=\mathfrak{c}$. In the Sacks model, however, the value of $\mathfrak{i}$ is small (namely $\aleph_{1}$ ), this fact is due to unpublished work of Shelah and Eisworth (for an indirect proof see [5]). In this paper, we present a direct proof of this assertion (see Theorem 29). Among other things, the analysis we offer leads to the construction of a co-analytic Sacks indestructible maximal independent family (see [4]).

More generally, in this paper, we study two ideals which are naturally associated to independent families. The first (see Definition 4) is characterized by a diagonalization property, which allows along finite support iterations of ccc posets, the length of which is of uncountable cofinality, to iteratively construct, and so adjoint to the initial ground model, a maximal independent family of arbitrary size. More precisely, given an independent family $\mathcal{A}$, one can construct an ideal $\mathcal{J}_{\mathcal{A}}$ and a ccc poset, to which we will refer as independence diagonalization poset, which adjoins a real $\sigma$ with the following two properties:

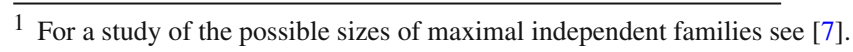


(1) $\{\sigma\} \cup \mathcal{A}$ is independent;

(2) for all $x \in[\omega]^{\omega} \backslash \mathcal{A}$ if $\mathcal{A} \cup\{x\}$ is independent, then $\mathcal{A} \cup\{x, \sigma\}$ is not independent.

Analogous weak ${ }^{2}$ diagonalization properties are well-known for maximal almost disjoint families (see [14]), maximal cofinitary groups (see [9]), and towers (see [8]). Thus along a finite support iterations of length $\lambda$ such that $\operatorname{cof}(\lambda)=\kappa>\omega$, one can iteratively adjoin a maximal independent family of size $\kappa$. The maximality of the iteratively constructed independent family is provided by property (2) and the wellknown fact that along finite support ccc iterations Cohen reals are added at stages of countable cofinalities.

Even though the diagonalization ideals are not unique, they all contain another ideal which is naturally associated to the independent families, the density independence ideal introduced in Lemma 11. The study of the density independence ideal originates in Shelah's proof of the consistency of $\mathfrak{i}<\mathfrak{u}$ in [15]. In fact, building upon the techniques developed in [15], we introduce a countably closed poset (see Definition 13), which naturally adjoins a Sacks indestructible (i.e. indestructible under the countable support iterations of Sacks forcing) maximal independent family (see Theorem 29).

A careful analysis of the construction and the relevant preservation properties shows that the Sacks indestructibility of the generic family $\mathcal{A}_{G}$ is captured by a combinatorial property, denoted $(\star)$ in the proof of Theorem 29 and the fact that the dual filter of $\operatorname{id}(\mathcal{A})$ is Ramsey. Property $(\star)$ expresses in particular the following maximality property of $\operatorname{id}(\mathcal{A})$ : for all $h \in \operatorname{FF}(\mathcal{A})$ and all $X \subseteq \omega$ such that $X \subseteq \mathcal{A}^{h}$, either the set $\mathcal{A}^{h} \backslash X$ is negligible and is in the ideal $\operatorname{id}(\mathcal{A})$, or there is an entire boolean combination contained in $\mathcal{A}^{h} \backslash X$. In Sect. 5, we proceed by comparing the ideals of diagonalization and density. We prove the minimality of the density independence ideal mentioned above. Namely, we show that for arbitrary independent family $\mathcal{A}$ and every diagonalization ideal $\mathcal{J}_{\mathcal{A}}, \operatorname{id}(\mathcal{A}) \subseteq \mathcal{J}_{\mathcal{A}}$ (see Lemma 31). We point out that the ideals are not necessarily equal (see Lemma 32 ) and that the density ideal is not necessarily a diagonalization ideal. However, for the following class of maximal independent families, these two types of ideals do coincide. We define an independent family $\mathcal{A}$ to be densely maximal (see Definition 33) if for every $X \in[\omega]^{\omega} \backslash \mathcal{A}$ and every $h \in \operatorname{FF}(\mathcal{A})$, there is $h^{\prime} \in \operatorname{FF}(\mathcal{A})$ for which either $X \cap \mathcal{A}^{h^{\prime}}$ or $\mathcal{A}^{h^{\prime}} \backslash X$ is finite. That is, a maximal independent family is densely maximal if examples of maximality occur densely along the tree of boolean combinations. We show that for infinite independent families, dense maximality is equivalent to property $(\star$ ) stated above (see Lemma 34 ) and that for a densely maximal independent family $\mathcal{A}$, the density ideal $\operatorname{id}(\mathcal{A})$ contains any ideal disjoint from the boolean hull of the family $\mathcal{A}$. The latter fact implies in particular, that for densely maximal independent families the density and diagonalization ideals coincide (see Corollary 36). Our proof of Theorem 29 implies that a sufficient condition for a densely maximal independent family to be preserved under the countable support iteration of Sacks forcing is that the dual ideal of its density ideal is generated by a Ramsey filter and the co-finite sets.

Finally, we show that for an arbitrary independent family $\mathcal{A}$, neither $\mathcal{J}_{\mathcal{A}}$, nor $\operatorname{id}(\mathcal{A})$ is maximal (see Proposition 38). We conclude by pointing out that Sacks indestructible

\footnotetext{
${ }^{2}$ For mad families, as well as towers, there is a natural strengthening of this property, leading to the preservation of mad families and towers along appropriate 2D-iterations, see respectively $[3,8]$.
} 
densely maximal independent families can be inductively constructed under $\mathrm{CH}$, by making some further general remarks and stating some open questions.

\section{Adjoining an independent real}

Lemma 3, as well Definition 5 and Lemma 6 can be found in [12].

Lemma 3 Let $\mathcal{A}$ be an independent family. Then there is an ideal $\mathcal{J}_{\mathcal{A}}$ on $\omega$ with the following properties:

(1) $\mathcal{J}_{\mathcal{A}} \cap\left\{\mathcal{A}^{h}: h \in \mathrm{FF}(\mathcal{A})\right\}=\emptyset$.

(2) For every $X \in[\omega]^{\omega}$ there is $h \in \mathrm{FF}(\mathcal{A})$ such that either $X \cap \mathcal{A}^{h}$ or $\mathcal{A}^{h} \backslash X$ belongs to $\mathcal{J}_{\mathcal{A}}$.

Proof Let $\left\{X_{\alpha}\right\}_{\alpha \in \mathfrak{c}}$ be an enumeration of $[\omega]^{\omega}$. Inductively, construct an increasing sequence of ideals $\overline{\mathcal{J}}=\left\langle\mathcal{J}_{\alpha}: \alpha \in \mathfrak{c}\right\rangle$ as follows. Let $\mathcal{J}_{0}=[\omega]^{<\omega}$ and suppose $\mathcal{J}_{\alpha}$ has been constructed. Consider $X_{\alpha}$. If there is $h \in \mathrm{FF}(\mathcal{A})$ and $Y \in \mathcal{J}_{\alpha}$ such that $\mathcal{A}^{h} \subseteq X_{\alpha} \cup Y$, then take $\mathcal{J}_{\alpha+1}=\mathcal{J}_{\alpha}$. Otherwise, define $\mathcal{J}_{\alpha+1}$ to be the ideal generated by $\mathcal{J}_{\alpha} \cup\left\{X_{\alpha}\right\}$. Finally, if $\alpha<\mathfrak{c}$ is a limit ordinal, put $\mathcal{J}_{\alpha}=\bigcup_{\beta<\alpha} \mathcal{J}_{\beta}$. Once this inductive construction finishes, we define $\mathcal{J}_{\mathcal{A}}$ as $\mathcal{J}_{\mathcal{A}}=\bigcup_{\alpha \in \mathfrak{c}} \mathcal{J}_{\alpha}$.

To verify property (1) above, suppose by way of contradiction that there is $h_{0} \in$ $\mathrm{FF}(\mathcal{A})$ such that $\mathcal{A}^{h_{0}} \in \mathcal{J}_{\mathcal{A}}$. Since $\mathcal{A}^{h_{0}}$ is infinite, $\mathcal{J}_{\mathcal{A}} \neq \mathcal{J}_{0}$. Take $\beta>0$ minimal such that $\mathcal{A}^{h_{0}} \in \mathcal{J}_{\beta}$. Then $\beta=\alpha+1$ is a successor, $\mathcal{A}^{h_{0}} \notin \mathcal{J}_{\alpha}$ and since $\mathcal{A}^{h_{0}} \in \mathcal{J}_{\alpha+1}$ we must have $\mathcal{J}_{\alpha} \neq \mathcal{J}_{\alpha+1}$. Then, by construction of $\mathcal{J}_{\alpha+1}$, for all $h \in \operatorname{FF}(\mathcal{A})$ and all $Y \in \mathcal{J}_{\alpha}, \mathcal{A}^{h} \nsubseteq Y \cup X_{\alpha}$. On the other hand, since $\mathcal{A}^{h_{0}} \in \mathcal{J}_{\alpha+1} \backslash \mathcal{J}_{\alpha}=\left\langle\mathcal{J}_{\alpha} \cup\left\{X_{\alpha}\right\}\right\rangle \backslash \mathcal{J}_{\alpha}$, there must be $Y_{0} \in \mathcal{J}_{\alpha}$ such that $\mathcal{A}^{h_{0}} \subseteq Y_{0} \cup X_{\alpha}$, which is clearly a contradiction.

To verify property (2), consider any $X \in[\omega]^{\omega}$. Again $X=X_{\alpha}$ for some minimal $\alpha<\mathfrak{c}$. If $\mathcal{J}_{\alpha}=\mathcal{J}_{\alpha+1}$ then there are $h^{\prime} \in \operatorname{FF}(\mathcal{A})$ and $Y \in \mathcal{J}_{\alpha}$ such that $\mathcal{A}^{h^{\prime}} \subseteq X_{\alpha} \cup Y$. But then, $\mathcal{A}^{h^{\prime}} \backslash X_{\alpha} \subseteq Y$, which implies that $\mathcal{A}^{h^{\prime}} \backslash X_{\alpha} \in \mathcal{J}_{\mathcal{A}}$. Otherwise, $X_{\alpha} \in \mathcal{J}_{\alpha+1}$ and so property (2) trivially holds, since for each $h \in \mathrm{FF}(\mathcal{A}), X_{\alpha} \cap \mathcal{A}^{h} \subseteq X_{\alpha} \in \mathcal{J}_{\mathcal{A}}$ and so, clearly it belongs to $\mathcal{J}_{\mathcal{A}}$.

Definition 4 Whenever $\mathcal{A}$ is an independent family and $\mathcal{J}_{\mathcal{A}}$ is an ideal satisfying properties (1) and (2) of Lemma 3, we say that $\mathcal{J}_{\mathcal{A}}$ is an independence diagonalization ideal associated to $\mathcal{A}$.

More properties of these ideals will be presented in the upcoming sections.

Note The ideal $\mathcal{J}_{\mathcal{A}}$ constructed above is not unique and depends on the chosen enumeration of $\mathcal{P}(\omega)$. Suppose for instance that $\mathcal{A}$ is not maximal and take $X_{0}$ to be a subset of $\omega$ such that for all boolean combinations $h \in \mathrm{FF}(\mathcal{A})$ the sets $\mathcal{A}^{h} \cap X$ and $\mathcal{A}^{h} \backslash X$ are infinite.

Now consider an enumeration of $[\omega]^{\omega}, \pi_{0}$ for which $\pi_{0}(0)=X_{0}$ and denote by $\mathcal{J}^{0}$ the ideal associated to the family $\mathcal{A}$ with respect to $\pi_{0}$ and $\mathcal{J}_{\alpha}^{0}$ its corresponding approximations for all $\alpha<\mathfrak{c}$. Then by the definition we get $X_{0} \notin \mathcal{J}_{1}^{0}$ (the first step in the inductive construction relative to $\pi_{0}$ ) because $\mathcal{A}^{h} \nsubseteq X_{0} \cup Y$ for all $h \in \operatorname{FF}(\mathcal{A})$ and $Y \in \mathcal{J}_{0}^{0}=[\omega]^{<\omega}$. In other words, $\mathcal{A}^{h} \nsubseteq^{*} X_{0}$ for all $h \in \operatorname{FF}(\mathcal{A})$. 
On the other hand, if $\pi_{1}$ is another enumeration of $[\omega]^{\omega}$ such that $\pi_{1}(0)=\mathcal{A}^{g} \backslash X_{0}$ for some $g \in \operatorname{FF}(\mathcal{A})$ and we denote $\mathcal{J}^{1}$ to be the ideal associated to $\mathcal{A}$ with respect to $\pi_{1}$. Likewise, denote $\mathcal{J}_{\alpha}^{1}$ to be the corresponding inductive approximations of $\mathcal{J}^{1}$, for all $\alpha<\mathfrak{c}$. Thus, we must have $\mathcal{A}^{g} \backslash X_{0} \in \mathcal{J}_{1}^{1}$. Given $h \in \operatorname{FF}(\mathcal{A}), \mathcal{A}^{h} \backslash\left(\mathcal{A}^{g} \backslash X\right)=$ $\left(\mathcal{A}^{h} \cap\left(\omega \backslash \mathcal{A}_{g}\right)\right) \cup\left(\mathcal{A}^{h} \cap X\right)$ and the latter set $\mathcal{A}^{h} \cap X_{0}$ is always infinite. But then, this implies that $X_{0} \notin \mathcal{J}^{1}$, because otherwise $\left(\mathcal{A}^{g} \backslash X_{0}\right) \cup X_{0}$ would also be an element of $\mathcal{J}^{1}$ and this contradicts that $\mathcal{A}^{g} \notin \mathcal{J}_{\mathcal{A}}^{1}$.

The poset below adjoins a new real to a given independent family, and furthermore weakly diagonalizes the ground model with respect to the independent family $\mathcal{A}$ as described in Lemma 6.

Definition 5 Let $\mathcal{A}$ be an independent family and let $\mathcal{J}_{\mathcal{A}}$ be an independence diagonalization ideal associated to it. The poset $\mathbb{B}\left(\mathcal{J}_{\mathcal{A}}\right)$ consists of all pairs $(s, E)$ where $s \in[\omega]^{<\omega}, E \in\left[\mathcal{J}_{\mathcal{A}}\right]^{<\omega}$ with extension relation defined as follows: $(t, F) \leq(s, E)$ if and only if $t \supseteq s, F \supseteq E$ and $(t \backslash s) \cap \bigcup E=\emptyset$.

Throughout the paper, unless otherwise specified, $V$ denotes the ground model. Note that the poset $\mathbb{B}\left(\mathcal{J}_{\mathcal{A}}\right)$ is $\sigma$-centered, so it preserves cardinals. Additionally it has the following weak diagonalization property:

Lemma 6 Let $G$ be a $\mathbb{B}\left(\mathcal{J}_{\mathcal{A}}\right)$ generic filter. Then $x_{G}:=\bigcup\{s: \exists F(s, F) \in G\}$ is an infinite subset of $\omega$ such that in $V[G], \mathcal{A} \cup\left\{x_{G}\right\}$ is independent, while for every $Y \in\left([\omega]^{\omega} \backslash \mathcal{A}\right) \cap V$, the family $\mathcal{A} \cup\left\{x_{G}, Y\right\}$ is not independent.

Proof First, to see that $\mathcal{A} \cup\left\{x_{G}\right\}$ is independent notice that given a condition $(s, F) \in$ $\mathbb{B}\left(\mathcal{J}_{\mathcal{A}}\right), h \in \mathrm{FF}(\mathcal{A})$ and $n \in \omega$, we can extend the condition $(s, F)$ to $(t, G)$ such that both $t \cap \mathcal{A}^{h}$ and $\bigcup G \cap \mathcal{A}^{h}$ have size greater than $n$ because $\mathcal{A}^{h}$ is an infinite set for which $\mathcal{A}^{h} \backslash \bigcup F$ is infinite.

Second, let $Y \in\left([\omega]^{\omega} \backslash \mathcal{A}\right) \cap V$. Without loss of generality assume that $\mathcal{A} \cup\{Y\}$ is independent and consider an arbitrary $(s, E) \in \mathbb{B}\left(\mathcal{J}_{\mathcal{A}}\right)$. By definition of $\mathcal{J}_{\mathcal{A}}$ there is $h^{\prime} \in \operatorname{FF}(\mathcal{A})$ such that either $Y \cap \mathcal{A}^{h^{\prime}}$ or $Y \backslash \mathcal{A}^{h^{\prime}}$ is in $\mathcal{J}_{\mathcal{A}}$. In the former case note that $\left(s, E \cup\left\{Y \cap \mathcal{A}^{h^{\prime}}\right\}\right)$ is an extension of $(s, E)$ forcing that $x_{G} \cap Y \subseteq s$, while in the second case $\left(s, E \cup\left\{Y \backslash \mathcal{A}^{h^{\prime}}\right\}\right)$ is an extension of $(s, E)$ forcing that $x_{G} \cap Y \backslash \mathcal{A}^{h^{\prime}} \subseteq s$.

As a corollary we obtain:

Theorem 7 (GCH) Let $\kappa<\lambda$ be regular uncountable cardinals. There is a ccc generic extension in which $\mathfrak{i}=\mathfrak{d}=\kappa<\mathfrak{c}=\lambda$.

Proof Let $\gamma$ be the ordinal product $\lambda \cdot \kappa$. Then $|\gamma|=\lambda$ and $\operatorname{cof}(\gamma)=\kappa$. Let $E$ be a cofinal subset of $\gamma$ of cardinality $\kappa$ consisting of successor ordinals. Define a finite support iteration $\left\langle\mathbb{P}_{\alpha}, \dot{\mathbb{Q}}_{\beta}: \alpha \leq \gamma, \beta<\gamma\right\rangle$ as follows. Suppose we have defined $\mathbb{P}_{\beta}$ for each $\beta<\alpha$, as well as an increasing sequence of $\left\{\mathcal{A}_{\beta}\right\}_{\beta<\alpha}$ of independent families, where $\mathcal{A}_{\beta} \in V^{\mathbb{P}_{\beta}}$. If $\alpha$ is a limit, define $\mathbb{P}_{\alpha}$ to be the finite support iteration of $\left\langle\mathbb{P}_{\beta}: \beta<\alpha\right\rangle$ and $\mathcal{A}_{\alpha}=\bigcup_{\beta<\alpha} \mathcal{A}_{\beta}$. Now, suppose $\alpha$ is a successor, $\alpha=\xi+1$.

If $\alpha \in E$, in $V^{\mathbb{P}_{\xi}}$ fix an independence diagonalization ideal $\mathcal{J}_{\xi}=\mathcal{J}_{\mathcal{A}_{\xi}}$ associated to the independent family $\mathcal{A}_{\xi}$ and take $\dot{\mathbb{Q}}_{\xi}$ to be a $\mathbb{P}_{\xi}$-name for $\mathbb{B}\left(\mathcal{J}_{\xi}\right)$. Let $x_{\xi}$ be the 
generic adjoined by $\mathbb{Q}_{\xi}$ over $V^{\mathbb{P}_{\xi}}$ and define $\mathcal{A}_{\alpha}=\mathcal{A}_{\xi} \cup\left\{x_{\xi}\right\}$. If $\alpha \notin E$, let $\dot{\mathbb{Q}}_{\alpha}$ be a $\mathbb{P}_{\alpha}$ name for the Cohen poset $\mathbb{C}, \mathcal{A}_{\alpha}=\mathcal{A}_{\xi}$. With this the inductive definition of $\mathbb{P}_{\gamma}$ is complete.

Recall that the iteration defined above is ccc. Also, since $\operatorname{cof}(\gamma)=\kappa$, the Cohen reals added along a cofinal in $\gamma$ sequence of length $\kappa$ form an unbounded family in $V^{\mathbb{P}_{\gamma}}$ and so $V^{\mathbb{P}_{\gamma}} \vDash \kappa \leq \mathfrak{d}$.

The family $\mathcal{A}=\mathcal{A}_{\gamma}$ is clearly independent, as the increasing union of independent families. Suppose there is $Y \in V^{\mathbb{P}_{\gamma}} \cap[\omega]^{\omega}$, which is not in $\mathcal{A}$, yet $\mathcal{A} \cup\{Y\}$ is independent. There is $\alpha=\xi+1 \in E$ such that $Y \in V^{\mathbb{P}_{\xi}}$. Then $V^{\mathbb{P}_{\xi}} \vDash \mathcal{A}_{\xi} \cup\{Y\}$ is independent and so by Lemma $6 V^{\mathbb{P}_{\alpha}} \vDash \mathcal{A}_{\alpha} \cup\{Y\}$ is not independent, which is a contradiction. Thus $V^{\mathbb{P}_{\gamma}} \vDash \mathcal{A}$ is maximal and so $V^{\mathbb{P}_{\gamma}} \vDash \kappa \leq \mathfrak{d} \leq \mathfrak{i} \leq \kappa$.

The following lemma shows that the increasing enumeration function of the generic real added by $\mathbb{B}\left(\mathcal{J}_{\mathcal{A}}\right)$ is unbounded over the ground model reals.

Lemma 8 Let $G$ be $\mathbb{B}\left(\mathcal{J}_{\mathcal{A}}\right)$ generic filter and let $\sigma_{G}$ be the enumerating increasing function of $x_{G}$. Then for all $g \in{ }^{\omega} \omega \cap V, V[G] \vDash \exists^{\infty} n\left(g(n)<\sigma_{G}(n)\right)$.

Proof It is sufficient to show that for each $n \in \omega$ and each $g \in{ }^{\omega} \omega \cap V$ the set

$$
D_{n}=\left\{(s, F): \exists m>n\left((s, F) \Vdash \sigma_{G}(m)>g(m)\right)\right\}
$$

is dense. Fix $n \in \omega,(s, F) \in \mathbb{B}\left(\mathcal{J}_{\mathcal{A}}\right)$. Let $K:=\omega \backslash(\bigcup F \cup s)$. Consider $K$ with its increasing enumeration and take an initial segment $\Delta_{0}$ of $K$ such that $s \cup \Delta_{0}$ is an initial segment of $K \cup s$ and $\left|s \cup \Delta_{0}\right|=m$ for some $m>n$. Note that $\left(s \cup \Delta_{0}, F\right)$ forces that the enumerating function of $s \cup \Delta_{0}$ is equal to $\sigma_{G}\left\lceil m\right.$. Now, take $\Delta_{1}=$ $\left(K \backslash \Delta_{0}\right) \cap(g(m)+1)$ and let $k^{*}=\min K \backslash\left(\Delta_{0} \cup \Delta_{1}\right)$. Then $q:=\left(s \cup \Delta_{0} \cup\left\{k^{*}\right\}, F \cup \Delta_{1}\right)$ extends $\left(s \cup \Delta_{0}, F\right)$ and forces $\sigma_{G}(m)=k^{*}$. Thus in particular, $q \leq(s, F)$ and $q \Vdash \sigma_{G}(m)>g(m)$.

A natural question to ask is whether the diagonalization poset also adds a dominating real. Below, we present a sufficient condition for $\mathbb{B}\left(\mathcal{J}_{\mathcal{A}}\right)$ to adjoin a dominating real and in Sects. 5 and 6 we will point out the existence of maximal independent families for which these conditions are satisfied.

Lemma 9 Let $\mathcal{A}$ be an independent family in $V$ and let $\mathcal{J}_{\mathcal{A}}$ be an independence diagonalization ideal associated to it. Suppose there is a family $\left\{X_{g}: g \in \omega^{\omega} \cap\right.$ $V$ and $g$ is increasing $\} \subseteq V \cap \mathcal{P}(\omega)$ such that for each $g$ :

(1) $X_{g} \subseteq[g(0), \omega)$;

(2) $\omega \backslash X_{g} \in \mathcal{J}_{\mathcal{A}}$;

(3) for all but finitely many $n,\left|X_{g} \cap[g(n), g(n+1))\right| \leq 1$.

Let $G$ be $\mathbb{B}\left(\mathcal{J}_{\mathcal{A}}\right)$ generic over $V$ and let $\sigma_{G}$ be the increasing enumerating of $x_{G}$. Then $\sigma_{G}$ dominates $\omega^{\omega} \cap V$.

Proof Let $g \in \omega^{\omega} \cap V,(s, F) \in \mathbb{B}\left(\mathcal{J}_{\mathcal{A}}\right)$ and $m$ minimal such that for all $n \geq m$,

$$
\left|X_{g} \cap[g(n), g(n+1))\right| \leq 1 \text { and } n \geq \max (s) .
$$


Fix $\Delta_{0} \subseteq K=\omega \backslash(\bigcup F \cup s)$ so that $s \cup \Delta_{0}$ is an initial segment of $K \cup s$ and $\left|s \cup \Delta_{0}\right|=m$. Let $u$ be the increasing enumeration of $\Delta_{0} \cup s$.

Thus $\left(s \cup \Delta_{0}, F\right) \Vdash u=\dot{\sigma}_{G}\left\lceil m\right.$ and putting $H=F \cup\left\{\omega \backslash X_{g},[0, g(m))\right\}$ (which by hypothesis is a finite subset of $\left.\mathcal{J}_{\mathcal{A}}\right)$, we obtain $\left(s \cup \Delta_{0}, H\right) \leq\left(s \cup \Delta_{0}, F\right)$ and $\left(s \cup \Delta_{0}, H\right) \Vdash \forall n \geq m\left(\dot{\sigma}_{G}(n) \notin F \cup\left\{\omega \backslash X_{g},[0, g(m))\right\}\right)$. In particular, $\left(s \cup \Delta_{0}, H\right) \Vdash$ $\forall n \geq m\left(\dot{\sigma}_{G}(n) \in X_{g} \cap[g(m), \omega)\right)$, which implies $\left(s \cup \Delta_{0}, H\right) \Vdash \forall n \geq m\left(\dot{\sigma}_{G}(n) \geq\right.$ $g(n))$.

Definition 10 A filter $\mathcal{F}$ on $\omega$ is a Ramsey, if for every partition $\left\{A_{n}: n \in \omega\right\}$ such that $\omega \backslash A_{n} \in \mathcal{F}$, there is a sequence $\left\{k_{n}: n \in \omega\right\} \in \mathcal{F}$ with $k_{n} \in A_{n}$, for all $n \in \omega$.

The lemma above suggests that ideals of the form $\mathcal{J}_{\mathcal{A}}$ such that the corresponding filters $\mathcal{F}_{\mathcal{A}}=\mathcal{P}(\omega) \backslash \mathcal{J}_{\mathcal{A}}$ are Ramsey are good candidates for domination. In Sect. 5, we will see that independent families the diagonalization ideal of which has the above property do exist.

\section{A generic maximal independent family}

In [15] Shelah constructs a maximal independent family which remains a witness to $\mathfrak{i}=\aleph_{1}$ in a model of $\mathfrak{u}=\aleph_{2}$. We show that, over a model of GCH for example, his construction naturally gives rise to the existence of a countably closed, $\aleph_{2}$-cc poset $\mathbb{P}$ which generically adjoins a maximal independent family, which as shown in the next section is Sacks indestructible.

Lemma 11 Let $\mathcal{A}$ be an independent family and let $\mathcal{D}(X)$ to be the set of all functions $h \in \operatorname{FF}(\mathcal{A})$ for which $X \cap \mathcal{A}^{h}$ is finite. Then

$$
\begin{aligned}
\operatorname{id}(\mathcal{A}) & =\left\{X \subseteq \omega: \forall h \in \mathrm{FF}(\mathcal{A}) \exists h^{\prime} \supseteq h\left(\mathcal{A}^{h^{\prime}} \cap X\right) \text { is finite }\right\} \\
& =\{X \subseteq \omega: \mathcal{D}(X) \text { is dense in } \operatorname{FF}(\mathcal{A})\}
\end{aligned}
$$

is an ideal on $\omega$, to which we refer as the independence density ideal associated to $\mathcal{A}$. Here when we say "dense" in $\operatorname{FF}(\mathcal{A})$, we mean dense respect to the inclusion relation.

(2) If $\mathcal{A}_{0}, \mathcal{A}_{1}$ are independent families such that $\mathcal{A}_{0} \subseteq \mathcal{A}_{1}$, then $\operatorname{id}\left(\mathcal{A}_{0}\right) \subseteq \operatorname{id}\left(\mathcal{A}_{1}\right)$.

Proof Straightforward.

Remark 12 If $\mathcal{A}$ is an infinite independent family, $X \subseteq \omega$, and $h \in \mathrm{FF}(\mathcal{A})$ is such that $\mathcal{A}^{h} \cap X$ is finite, then there is $h_{1} \in \mathrm{FF}(\mathcal{A})$ such that $\bar{h}_{1} \supseteq h$ and $\mathcal{A}^{h_{1}} \cap X=\emptyset$.

Definition 13 Let $\mathbb{P}$ be the poset of all pairs $(\mathcal{A}, A)$ where $\mathcal{A}$ is a countable independent family, $A \in[\omega]^{\omega}$ such that for all $h \in \mathrm{FF}(\mathcal{A})$ the set $\mathcal{A}^{h} \cap A$ is infinite. The extension relation on $\mathbb{P}$ is given by: $(\mathcal{B}, B) \leq(\mathcal{A}, A)$ if and only if $\mathcal{B} \supseteq \mathcal{A}$ and $B \subseteq \subseteq^{*} A$.

The following shows that the sets in $\operatorname{id}(\mathcal{A})$ are indeed negligible sets. 
Lemma 14 Let $(\mathcal{A}, A) \in \mathbb{P}$ and let $X \in \operatorname{id}(\mathcal{A})$. Then $(\mathcal{A}, A \backslash X) \in \mathbb{P}$.

Proof Sufficient to show that for each $h \in \mathrm{FF}(\mathcal{A})$, the set $\mathcal{A}^{h} \cap(A \backslash X)$ is infinite. Fix $h \in \mathrm{FF}(\mathcal{A})$. Since $X \in \operatorname{id}(\mathcal{A})$, there is $h^{\prime} \supseteq h, h^{\prime} \in \mathrm{FF}(\mathcal{A})$ such that $\mathcal{A}^{h^{\prime}} \cap X=\emptyset$, which implies $\mathcal{A}^{h^{\prime}} \subseteq \omega \backslash X$. Then $\mathcal{A}^{h^{\prime}} \cap(\bar{A} \backslash X)=\mathcal{A}^{h^{\prime}} \cap A$, and so $\mathcal{A}^{h^{\prime}} \cap(A \backslash X)$ is infinite. However $\mathcal{A}^{\bar{h}^{\prime}} \cap(A \backslash X) \subseteq \mathcal{A}^{h} \cap(A \backslash X)$, which implies that $\mathcal{A}^{h} \cap(A \backslash X)$ is also infinite. Thus $(\mathcal{A}, A \backslash X)$ is indeed a condition.

Another straightforward observation following the above Lemma is that for each $X \in \operatorname{id}(\mathcal{A})$, the set of conditions $(\mathcal{B}, B)$ in $\mathbb{P}$ such that $X \cap B=\emptyset$ is dense below $(\mathcal{A}, A)$. The poset $\mathbb{P}$ has the following properties (see also [15, Claim 2.2]).

Proposition 15 The poset $\mathbb{P}$ is $\sigma$-closed. Furthermore, if $\mathrm{CH}$ holds and $2^{\aleph_{1}}=\aleph_{2}$, then $\mathbb{P}$ is $\aleph_{2}-c c$.

Proof To verify that $\mathbb{P}$ is $\sigma$-closed, consider a decreasing chain of conditions, $\left\{\left(\mathcal{A}_{n}, A_{n}\right): n \in \omega\right\}$. Without loss of generality, we can assume that for all $n \in \omega$, $A_{n+1} \subseteq A_{n}$. Let $\mathcal{A}=\bigcup_{n \in \omega} \mathcal{A}_{n}$. Now, for each $n$, let $\left\{h_{n, l}: l \in \omega\right\}$ enumerate $\mathrm{FF}\left(\mathcal{A}_{n}\right)$. If $m \leq n$, then $\mathcal{A}_{m} \subseteq \mathcal{A}_{n}$ and so $\mathrm{FF}\left(\mathcal{A}_{m}\right) \subseteq \mathrm{FF}\left(\mathcal{A}_{n}\right)$. Now, for each $n \in \omega$ pick $\left\{k_{n, m, l}: m \leq n, l \leq n\right\}$ such that $k_{n, m, l} \in A_{n} \cap \mathcal{A}_{n}^{h_{m, l}}$.

Finally, let $A=\left\{k_{n, m, l}: n \in \omega, m \leq n, l \leq n\right\}$. Note that $A \backslash A_{n}=\left\{k_{i, m, l}: i<\right.$ $n, m \leq i, l \leq i\}$ is finite and so $A$ is a pseudo-intersection of the $A_{n}$ 's meeting each $\mathcal{A}^{h}$ for $h \in \overline{\operatorname{FF}}(\mathcal{A})$ on an infinite set.

Assuming $\mathrm{CH}$ given a set of $\aleph_{2}$-many conditions in $\mathbb{P}, X=\left\{\left(\mathcal{A}_{\alpha}, A_{\alpha}\right): \alpha<\aleph_{2}\right\}$, we can assume that $A_{\alpha}=A_{\beta}$ for all $\alpha, \beta<\aleph_{2}$. If $2^{\aleph_{1}}=\aleph_{2}$, then there are just $\aleph_{1}$-many countable subsets of $\aleph_{1}$. So there must be an uncountable set of compatible conditions in $X$.

For convenience, we will introduce some terminology:

Definition 16 Let $\mathcal{E}$ be a partition of $\omega$ and $A \in[\omega]^{\omega}$. We will say that $\chi(\mathcal{E}, A)$ holds, if either there is $E \in \mathcal{E}$ such that $A \subseteq E$, or for each $E \in \mathcal{E}$ we have $|E \cap A| \leq 1$. Whenever the latter option holds, we will say that $A$ is a semiselector for $\mathcal{E}$.

Lemma 17 If $(\mathcal{A}, A) \in \mathbb{P}$, then there is $B \notin \mathcal{A}, B \subseteq A$ such that $(\mathcal{A} \cup\{B\}, A) \leq$ $(\mathcal{A}, A)$.

Proof Let $\left\{h_{n}: n \in \omega\right\}$ be a fixed enumeration of $\operatorname{FF}(\mathcal{A})$. Since $\mathcal{A}^{h_{0}} \cap A$ is infinite, we can find distinct $k_{0,0}, k_{0,1}$ in it. Proceed inductively. Suppose we have defined $\left\{k_{i, j}: i \in n, j \in 2\right\}$ all distinct. Since $\mathcal{A}^{h_{n}} \cap A$ is infinite, we can find distinct $k_{n, 0}$ and $k_{n, 1}$ in $\mathcal{A}^{h_{n}} \cap A \backslash\left\{k_{i, j}: i \in n, j \in 2\right\}$. Finally, take $B=\left\{k_{i, 0}: i \in \omega\right\}$. Clearly, $B \subseteq A$. To see that $\mathcal{A} \cup\{B\}$ is independent, consider an arbitrary $h \in \operatorname{FF}(\mathcal{A})$. Note that $\exists^{\infty} m\left(h \subseteq h_{m}\right)$. But then for infinitely many $m$, we have $k_{m, 0} \in \mathcal{A}^{h_{m}} \cap B \subseteq \mathcal{A}^{h} \cap B$ and $k_{m, 1} \in \mathcal{A}^{h_{m}} \backslash B \subseteq \mathcal{A}^{h} \backslash B$, which implies that both $\mathcal{A}^{h} \cap B$ and $\mathcal{A}^{h} \backslash B$ are infinite.

Lemma 18 If $(\mathcal{A}, A) \in \mathbb{P}, \mathcal{E}$ is a partition of $\omega$ and $h^{0} \in \mathrm{FF}(\mathcal{A})$, then there exist $h^{1} \supseteq h^{0}, B \subseteq A$ such that $(\mathcal{A}, B) \leq(\mathcal{A}, A)$ and $\chi\left(\mathcal{E}, \mathcal{A}^{h_{1}} \cap B\right)$. Moreover, if $|E|<\omega$ for each $E \in \mathcal{E}$, then $\mathcal{A}^{h^{1}} \cap B$ is a semiselector for $\mathcal{E}$. 
Proof Let $\left\{h_{n}: n \in \omega\right\}$ enumerate $\left\{h \in \mathrm{FF}(\mathcal{A}): h^{0} \subseteq h\right\}$, where $h_{0}$ is the given finite function $h^{0}$. Suppose we can inductively construct a sequence $\left\{k_{n}: n \in \omega\right\}$ such that for each $n, k_{n} \in\left(\mathcal{A}^{h_{n}} \cap A\right) \backslash\left(\bigcup\left\{\mathcal{E}\left(k_{l}\right): l<n\right\}\right)$, where $\mathcal{E}\left(k_{l}\right)$ is the unique $E \in \mathcal{E}$ such that $k_{l} \in E$. Then, take $B=\left\{k_{n}: n \in \omega\right\} \cup\left(A \backslash \mathcal{A}^{h^{0}}\right)$ and notice that $B \subseteq A$; we claim that $\mathcal{A} \cup\{B\}$ is independent. Indeed: Fix $h \in \operatorname{FF}(\mathcal{A})$. If $h$ and $h^{0}$ are compatible, consider $h^{\prime}=h \cup h^{0}$. Then for infinitely many $m$, we have $k_{m} \in \mathcal{A}^{h_{m}} \subseteq \mathcal{A}^{h^{\prime}}$ and so $\mathcal{A}^{h} \cap B$ is infinite. If $h$ and $h^{0}$ are incompatible, then there is $C \in \operatorname{dom}(h) \cap \operatorname{dom}\left(h^{0}\right)$ such that $h(C) \neq h^{0}(C)$. Without loss of generality, assume $h(C)=0$. Then $\mathcal{A}^{h}=\mathcal{A}^{h^{\prime}} \cap C$, where $h^{\prime}=h\lceil\operatorname{dom}(h) \backslash\{C\}$. On the other hand, $\mathcal{A}^{h^{0}} \subseteq \omega \backslash C$ and so $A \backslash \mathcal{A}^{h^{0}} \supseteq A \cap C$. Therefore

$$
\mathcal{A}^{h} \cap B \supseteq\left(\mathcal{A}^{h^{\prime}} \cap C\right) \cap A \cap C \supseteq \mathcal{A}^{h} \cap A .
$$

However $(\mathcal{A}, A)$ is a condition and so $\mathcal{A}^{h} \cap A$ is infinite. Thus $\mathcal{A}^{h} \cap B$ is also infinite.

Otherwise, we can finite a finite sequence $\left\{k_{l}: l<n\right\}$ such that for each $l<n$, $k_{l} \in\left(\mathcal{A}^{h_{l}} \cap A\right) \backslash \bigcup\left\{\mathcal{E}\left(k_{j}\right): j<l\right\}$, but $\mathcal{A}^{h_{n}} \cap A \subseteq \bigcup\left\{\mathcal{E}\left(k_{l}\right): l<n\right\}$. By induction on $l \leq n$, try to find $h_{n, l} \in \mathrm{FF}(\mathcal{A})$ such that $h_{n, 0}=h_{n}, h_{n, l} \subseteq h_{n, l^{\prime}}$ for $l<l^{\prime}$ and $A \cap \overline{\mathcal{A}}^{h_{n, l+1}} \cap \mathcal{E}\left(k_{l}\right)$ is finite. If we succeed to do this for each $l<n$, then $\mathcal{A}^{h_{n, n}} \cap A$ will be covered by a finite set, which is a contradiction. Therefore there is $l$ such that $0 \leq l<n$ and for each $h \supseteq h_{n, l}$ the set $\mathcal{A}^{h} \cap A \cap \mathcal{E}\left(k_{l}\right)$ is infinite. Take $h^{1}=h_{n, l}$, $B=\left(A \cap \mathcal{A}^{h^{1}} \cap \mathcal{E}\left(k_{l}\right)\right) \cup A \backslash \mathcal{A}^{h^{1}}$. Then clearly $\mathcal{A}^{h^{1}} \cap B \subseteq \mathcal{E}\left(k_{l}\right)$. It remains to show that $\mathcal{A} \cap\{B\}$ is independent. Fix $h \in \operatorname{FF}(\mathcal{A})$. If $h$ is compatible with $h^{1}$, then $h^{\prime}=h^{1} \cup h$ extends $h^{1}$ and so by the choice of $h_{n, l}, \mathcal{A}^{h^{\prime}} \cap B=\mathcal{A}^{h^{\prime}} \cap A \cap \mathcal{E}\left(k_{l}\right)$ is infinite. If $h$ and $h^{1}$ are incompatible, then there is $C \in \operatorname{dom}(h) \cap \operatorname{dom}\left(h^{1}\right)$ such that $h(C) \neq h^{1}(C)$. Without loss of generality, $h(C)=0$. Then $\mathcal{A}^{h} \cap B \supseteq A \cap C$, since $A \backslash \mathcal{A}^{h^{1}} \supseteq A \cap C$, and so $\mathcal{A}^{h} \cap B$ is infinite.

Corollary 19 Let $\mathcal{E}$ be a partition of $\omega$.

(1) The set of $(\mathcal{A}, A) \in \mathbb{P}$ such that for all $h \in \mathrm{FF}(\mathcal{A}) \exists h^{\prime} \in \mathrm{FF}(\mathcal{A})$ such that $\chi\left(\mathcal{E}, \mathcal{A}^{h^{\prime}}\right)$ is dense in $\mathbb{P}$.

(2) If $\mathcal{E}$ is a partition into finite sets, then the set of $(\mathcal{A}, A) \in \mathbb{P}$ such that $A$ is a semiselector for $\mathcal{E}$ is dense in $\mathbb{P}$.

Proof (1) Given $(\mathcal{A}, A)$ and an $h^{0} \in \mathrm{FF}(\mathcal{A})$, by Lemma 18 we can find $h^{1} \supseteq h^{0}$, $B \subseteq A$ such that $(\mathcal{A}, B) \leq(\mathcal{A}, A)$ and $\chi\left(\mathcal{E}, \mathcal{A}^{h_{1}} \cap B\right)$. Now, by Lemma 17 , there is $B^{\prime} \subseteq B$ such that $\left(\mathcal{A} \cup\left\{B^{\prime}\right\}, B\right) \leq(\mathcal{A}, B)$ and so $h_{2}=h_{1} \cup\left\{\left(B^{\prime}, 0\right)\right\}$ extends $h_{0}$, and $\chi\left(\mathcal{E}, \mathcal{A}_{1}^{h_{2}}\right)$ where $\mathcal{A}_{1}=\mathcal{A} \cup\left\{B^{\prime}\right\}$. Repeat countably many times the above argument, to obtain a countable decreasing sequence of conditions $\left\{\left(\mathcal{A}_{n}, A_{n}\right)\right\}_{n \in \omega}$ such that $\mathcal{A}_{\omega}=\bigcup \mathcal{A}_{n}$ is closed with respect to the above property and take $A_{\omega}$ to be a pseudointersection of the $A_{n}$ 's. Then $\left(\mathcal{A}_{\omega}, A_{\omega}\right)$ is as desired.

(2) Let $\left\{h_{n}: n \in \omega\right\}$ enumerate $\operatorname{FF}(\mathcal{A})$. Inductively construct a sequence $\left\{k_{n, i}: n \in\right.$ $\omega, i \in 2\}$ of distinct numbers such that for each $n, i, k_{n, i} \in\left(\mathcal{A}^{h_{n}} \cap A\right) \backslash\left(\bigcup\left\{\mathcal{E}\left(k_{l, i}\right)\right.\right.$ : $l<n, i \in 2\})$, where $\mathcal{E}\left(k_{l, i}\right)$ is the unique $E \in \mathcal{E}$ such that $k_{l, i} \in E$. Then, take $B=\left\{k_{n, 0}: n \in \omega\right\}$. Then $B \subseteq A$ and we claim that $\mathcal{A} \cup\{B\}$ is independent. Indeed. Fix $h \in \operatorname{FF}(\mathcal{A})$. Then for infinitely many $m$, we have $k_{m, i} \in \mathcal{A}^{h_{m}} \subseteq \mathcal{A}^{h^{\prime}}$, and so both $\mathcal{A}^{h} \cap B$, as well as $\mathcal{A}^{h} \backslash B$ are infinite. 
Corollary 20 Let $G$ be $\mathbb{P}$-generic over V. Then $\mathcal{A}_{G}=\bigcup\left\{\mathcal{A}: \exists A \in[\omega]^{\omega}\right.$ with $(\mathcal{A}, A) \in$ $G\}$ is a maximal independent family.

Proof Suppose $X \in[\omega]^{\omega} \backslash \mathcal{A}_{G}$ and $\mathcal{A}_{G} \cup\{X\}$ is independent. Pick $(\mathcal{A}, A) \in G$ such that

$$
(\mathcal{A}, A) \Vdash “ \mathcal{A}_{G} \cup\{X\} \text { is independent and } X \notin \mathcal{A}_{G}^{\prime \prime} \text {. }
$$

Consider $(\mathcal{A}, A)$. If for each $h \in \mathrm{FF}(\mathcal{A}),\left|\mathcal{A}^{h} \cap X \cap A\right|=\left|\mathcal{A}^{h} \cap A \cap X^{c}\right|=\omega$, then $(\mathcal{A} \cup\{X\}, A) \leq(\mathcal{A}, A)$ and $(\mathcal{A} \cup\{X\}, A) \Vdash$ " $X \in \mathcal{A}_{G}^{\prime \prime}$, which is a contradiction. Otherwise, there is $h \in \operatorname{FF}(\mathcal{A})$ such that $\left|\mathcal{A}^{h} \cap A \cap X\right|<\omega$ or $\left|\mathcal{A} \cap A \cap X^{c}\right|<\omega$. However, by Lemma 17 there is $B \notin \mathcal{A}$ such that $B \subseteq A$ and $(\mathcal{A} \cup\{B\}, A) \leq(\mathcal{A}, A)$. But then,

$$
(\mathcal{A} \cup\{B\}, A) \Vdash “ \exists h \in \operatorname{FF}\left(\mathcal{A}_{G}\right) \text { such that } \mathcal{A}_{G}^{h} \cap X \text { or } \mathcal{A}_{G}^{h} \backslash X \text { is finite". }
$$

Therefore $(\mathcal{A} \cup\{B\}, A) \Vdash$ " $\mathcal{A}_{G} \cup\{X\}$ is not independent", which is a contradiction.

Thus, forcing with $\mathbb{P}$ over a model of $\mathrm{CH}$ and $2^{\aleph_{1}}=\aleph_{2}$ adjoins a maximal independent family which is necessarily of size $\aleph_{1}$ as the poset does not add any new reals.

Lemma $21 \Vdash_{\mathbb{P}} \operatorname{id}\left(\mathcal{A}_{G}\right)=\bigcup\{\operatorname{id}(\mathcal{A}): \exists \mathcal{A}(\mathcal{A}, A) \in G\}$.

Proof The property follows from the countable closure of $\mathbb{P}$. Indeed, suppose there is $p=(\mathcal{A}, A) \in \mathbb{P}$ and $X \in[\omega]^{\omega} \cap V$ such that

$$
p \Vdash X \in \operatorname{id}\left(\mathcal{A}_{G}\right) \backslash(\bigcup\{\operatorname{id}(\mathcal{A}): \exists A(\mathcal{A}, A) \in G\}) .
$$

Consider $(\mathcal{A}, A), X$ and let $h \in \operatorname{FF}(\mathcal{A})$. Then

$$
p \Vdash \exists h^{\prime} \in \operatorname{FF}\left(\mathcal{A}_{G}\right)\left(h \subseteq h^{\prime} \text { and } \mathcal{A}^{h^{\prime}} \cap X=\emptyset\right) .
$$

Thus, there is $\left(\mathcal{A}^{\prime}, A^{\prime}\right) \in G$ extending $(\mathcal{A}, A)$ such that $h^{\prime} \in \mathrm{FF}\left(\mathcal{A}^{\prime}\right), h^{\prime} \supseteq h$ and $\mathcal{A}^{h^{\prime}} \cap X=\emptyset$. Proceed inductively to construct a decreasing sequence $\left\{\left(\mathcal{A}_{n}, A_{n}\right)\right\}$ of conditions below $p$, such that if $\mathcal{A}_{\omega}=\bigcup_{n \in \omega} \mathcal{A}_{n}$, then for all $h \in \operatorname{FF}\left(\mathcal{A}_{\omega}\right)$ there is $h^{\prime} \in \operatorname{FF}\left(\mathcal{A}_{\omega}\right)$ such that $h^{\prime} \supseteq h$ and $\mathcal{A}^{h^{\prime}} \cap X=\emptyset$. Thus $X \in \operatorname{id}\left(\mathcal{A}_{\omega}\right)$. Take $A_{\omega}$ to be any pseudointersection of $\left\{A_{n}\right\}_{n \in \omega}$ and let $p^{\prime}:=\left(\mathcal{A}_{\omega}, A_{\omega}\right)$. Then $p^{\prime} \leq p$ and

$$
p^{\prime} \Vdash X \in \bigcup\{\operatorname{id}(\mathcal{A}): \exists A(\mathcal{A}, A) \in G\},
$$

which is a contradiction.

Corollary 22 Let $G$ be a $\mathbb{P}$-generic filter. Then in $V[G]$ the density independence ideal $\operatorname{id}\left(\mathcal{A}_{G}\right)$ is generated by $\{\omega \backslash A: \exists \mathcal{A}(\mathcal{A}, A) \in G\}$. That is $\Vdash_{\mathbb{P}} \operatorname{id}\left(\mathcal{A}_{G}\right)=\langle\{\omega \backslash A:$ $\exists \mathcal{A}(\mathcal{A}, A) \in G\}\rangle$. 
Proof Let $G$ be a $\mathbb{P}$ generic filter. The lemma above gives us that in the generic extension $V[G], \operatorname{id}\left(\mathcal{A}_{G}\right)=\bigcup\{\operatorname{id}(\mathcal{A}): \exists \mathcal{A}(\mathcal{A}, A) \in G\}$, also let $\mathcal{I}_{G}$ to be the ideal generated by $\{\omega \backslash A: \exists \mathcal{A}(\mathcal{A}, A) \in G\}$ in the extension. First we show that $\operatorname{id}\left(\mathcal{A}_{G}\right) \subseteq$ $\mathcal{I}_{G}$. Let $X \in \operatorname{id}\left(\mathcal{A}_{G}\right)$. Thus there is $(\mathcal{A}, A) \in G$ such that $X \in \operatorname{id}(\mathcal{A})$. However $D_{X}=\{(\mathcal{B}, B): X \cap B=\emptyset\}$ is dense below $(\mathcal{A}, A)$ and so there is $(\mathcal{B}, B) \in G$ such that $X \cap B=\emptyset$. That is, $X \subseteq \omega \backslash B$ and so $X \in \mathcal{I}_{G}$.

To show that $\mathcal{I}_{G} \subseteq \operatorname{id}\left(\mathcal{A}_{G}\right)$, consider any $X \in \mathcal{I}_{G}$. Then there is a finite set of conditions $\left\{\left(\mathcal{A}_{i}, A_{i}\right)\right\}_{i \in n}$ in $G$ such that $X \subseteq \bigcup_{i \in n} \omega \backslash A_{i}=\omega \backslash \bigcap_{i \in n} A_{i}$. Note that $(\mathcal{B}, B) \in G$, where $(\mathcal{B}, B)=\left(\bigcup_{i \in n} \mathcal{A}_{i}, \bigcap_{i \in n} A_{i}\right)$. Thus $X \subseteq \omega \backslash B$. Fix any $h \in \operatorname{FF}\left(\mathcal{A}_{G}\right)$. Then, there is $(\mathcal{C}, C) \in G$ such that $h \in \operatorname{FF}(\mathcal{C})$. Without loss of generality $(\mathcal{C}, C) \leq(\mathcal{B}, B)$. Since the set $D_{B}=\left\{\left(\mathcal{C}^{\prime}, C^{\prime}\right): \exists Y \in \mathcal{C}(Y \subseteq B)\right\}$ is dense below $(\mathcal{B}, B)$, there is $\left(\mathcal{C}^{\prime}, C^{\prime}\right) \in G$ such that for some $Y \in \mathcal{C}^{\prime}, Y \subseteq B$. Then $h^{\prime}=h \cup\{(Y, 0)\} \in \operatorname{FF}\left(\mathcal{A}_{G}\right)$ and $\mathcal{A}_{G}^{h^{\prime}} \cap X=\emptyset$. Thus $X \in \operatorname{id}\left(\mathcal{A}_{G}\right)$.

Definition 23 (1) We say that a family $\mathcal{F} \subseteq[\omega]^{\omega}$ is a $Q$-set if for every partition $\mathcal{E}$ of $\omega$ into finite sets, there is $A \in \mathcal{F}$ such that $A$ is a semiselector for $\mathcal{E}$.

(2) A filter $\mathcal{F}$ on $\omega$ is said to be a $P$-set, if every countable subfamily of $\mathcal{F}$ has a common pseudointersection in $\mathcal{F}^{3}$

(3) A filter $\mathcal{F}$ on $\omega$ is a Ramsey, if for every partition $\left\{A_{n}: n \in \omega\right\}$ such that $\omega \backslash A_{n} \in \mathcal{F}$, there is a sequence $\left\{k_{n}: n \in \omega\right\} \in \mathcal{F}$ with $k_{n} \in A_{n}$, for all $n \in \omega$.

Lemma 24 Let $G$ be $\mathbb{P}$-generic, let $\mathcal{F}_{G}^{0}=\{A: \exists \mathcal{A}(A, \mathcal{A}) \in G\}$. Then $\mathcal{F}_{G}^{0}$ is a $Q$-set and the filter $\mathcal{F}_{G}$ generated by $\mathcal{F}_{G}^{0}$ and the co-finite sets is a $P$-set. Thus, in particular $\mathcal{F}_{G}$ is Ramsey.

Proof By Corollary $18 \mathcal{F}_{G}^{0}$ is a $Q$-set. Indeed, if $\mathcal{E}$ be a partition of $\omega$ into finite sets in $V[G]$, then $\mathcal{E}$ is a ground model set and so there is $(\mathcal{A}, A) \in G$ such that $A$ is a semiselector for $\mathcal{E}$. Clearly $A \in \mathcal{F}_{G}^{0}$.

To see that $\mathcal{F}_{G}$ is closed under finite intersections, consider arbitrary compatible conditions $\left(\mathcal{A}_{1}, A_{1}\right)$ and $\left(\mathcal{A}_{2}, A_{2}\right)$ in $\mathbb{P}$. Let $(\mathcal{C}, C)$ be their common extension in $G$. Then $\mathcal{C} \supseteq \mathcal{A}_{1} \cup \mathcal{A}_{2}$ and $C \subseteq \subseteq^{*} A_{1} \cap A_{2}$. Thus there is a finite $K$ such that $C \backslash K \subseteq A_{1} \cap A_{2}$. Thus if $A_{1}, A_{2}$ are from $\mathcal{F}_{G}^{0}$, then one can find $C \in \mathcal{F}_{G}$ as above, and a finite $K$ such that $A_{1} \cap A_{2}$ is a superset of $C \backslash K$, and so $A_{1} \cap A_{2} \in \mathcal{F}_{G}$.

It remains to show that every countable subfamily of $\mathcal{F}_{G}$ has a common pseudointersection in $\mathcal{F}_{G}$. Note that it is sufficient to show that $\Vdash\left(\mathcal{F}_{G}^{0}\right.$ is a $P$-set $)$. Suppose not. Then there is a condition $p \in \mathbb{P}$ such that

$$
p \Vdash \exists \mathcal{H}^{\prime} \in\left[\mathcal{F}_{G}^{0}\right]^{\omega} \text { s.t. } \forall F \in \mathcal{F}_{G}^{0} \exists H \in \mathcal{H}^{\prime}\left(F \nsubseteq^{*} H\right) .
$$

Fix $G$ a $\mathbb{P}$-generic filter such that $p \in G$. Since $\mathbb{P}$ is countably closed, we can find $\mathcal{H}^{\prime} \in V$ witnessing the above property. Thus $\mathcal{H}^{\prime}=\left\{\left(\mathcal{A}_{n}, A_{n}\right)\right\}$ where each $\left(\mathcal{A}_{n}, A_{n}\right) \in$ $G$. Without loss of generality $\left(\mathcal{A}_{0}, A_{0}\right)$ extends $p$ and $\left(\mathcal{A}_{n+1}, A_{n+1}\right) \leq\left(\mathcal{A}_{n}, A_{n}\right)$ for each $n$. Take $q=\left(\mathcal{A}_{\omega}, A_{\omega}\right)$ to be a common extension of the elements in $\mathcal{H}^{\prime}$. Then $q \leq p$ and $q$ forces that $A_{\omega} \in \mathcal{F}_{G}^{0}$ is a common pseudointersection of the sets in $\mathcal{H}^{\prime}$, which is a contradiction.

\footnotetext{
${ }^{3}$ The names of $P$-set and $Q$-set come from the usual terminology for $P$-points and $Q$-points.
} 
Corollary 25 Let $G$ be $\mathbb{P}$-generic. Then the dual filter fil $(\mathcal{A})$ of the density independence ideal $\operatorname{id}\left(\mathcal{A}_{G}\right)$ is generated by $\mathcal{F}_{G}^{0}$ and the co-finite sets, and so $\operatorname{fil}\left(\mathcal{A}_{G}\right)=\mathcal{F}_{G}$.

Proof Note that $\operatorname{id}\left(\mathcal{A}_{G}\right)$ is generated by $\{\omega \backslash A: \exists \mathcal{A}(\mathcal{A}, A) \in G\}$ and the finite sets.

\section{Sacks indestructibility}

In the following, we show that the above generic maximal independent family is Sacks indestructible. The existence of a Sacks indestructible maximal independent family is known (see for example [2] and [5]), however to the best knowledge of the authors a proof of its existence prior to the current work did not appear in the literature. Throughout the Section let $V$ denote the ground model, while $V_{0}=V^{\mathbb{P}}$. In addition, we assume that $V$ is a model of $\mathrm{CH}$ and $2^{\aleph_{1}}=\aleph_{2}$. Since $\mathbb{P}$ is countably closed, we clearly have $V \cap[\omega]^{\omega}=V_{0} \cap[\omega]^{\omega}$. By $\mathcal{A}$ we denote the generic independent family obtained in the previous section.

Lemma 26 Let $\mathbb{S}$ be Sacks forcing. Then for each $X \in \operatorname{id}(\mathcal{A})^{V_{0}^{\mathbb{S}}}$ there exists $Y \in$ $\operatorname{id}(\mathcal{A})^{V_{0}}$ such that $X \subseteq Y$.

Thus the density independence ideal of $\mathcal{A}$ in $V_{0}^{\mathbb{S}}$ has the same generating set as the density independence ideal of $\mathcal{A}$ in $V_{0}$.

Proof Recall first that in Lemma 11, the set $\mathcal{D}(X)$ was defined and one can characterize the density ideal using it. Namely, $\operatorname{id}(\mathcal{A})=\{X \subseteq \omega: \mathcal{D}(X)$ is dense in $\operatorname{FF}(\mathcal{A})\}$.

We shall then prove that if $\dot{\mathcal{D}}$ is a $\mathbb{S}$-name for an open dense subset of $\omega^{<\omega}$, then there exists $\mathcal{D}^{\prime} \in V_{0}$, an open dense subset of $\omega^{<\omega}$, such that there is a condition $S \in \mathbb{S}$ forcing $\mathcal{D}^{\prime} \subseteq \dot{\mathcal{D}}$. Note that this implies the result because given $X \in(\operatorname{id}(\mathcal{A}))^{V_{0}^{\mathbb{S}}}$ we get $\mathcal{D}(X)$ is dense in $\operatorname{FF}(\mathcal{A})$ which can be put in correspondence with $\omega^{<\omega}$ and so, we can find $\mathcal{D}^{\prime} \in V_{0}, \mathcal{D}^{\prime} \subseteq \mathcal{D}(X)$ open dense. Hence $Y=\bigcap_{h \in \mathcal{D}^{\prime}}\left(\omega \backslash \mathcal{A}^{h}\right) \in V_{0}$ and $Y \in \operatorname{id}(\mathcal{A})^{V_{0}}$.

Let $T \in \mathbb{S}$ be a condition forcing $\dot{\mathcal{D}} \subseteq \omega^{<\omega}$ is open dense and $\left\langle s_{n}: n \in \omega\right\rangle$ be an enumeration of $\omega^{<\omega}$. Inductively, we construct a fusion sequence $\left\langle S_{n}: n \in \omega\right\rangle$ in $\mathbb{S}$ and a sequence $\left\langle B_{n}: n \in \omega\right\rangle$ of ground model objects (in $V_{0}$ ) such that $B_{n} \subseteq \omega^{<\omega}$ and the following is satisfied:

- For every $n \in \omega, S_{n+1} \leq_{n} S_{n}$. Recall this means, $S_{n+1} \leq S_{n}$ and $\operatorname{split}_{n}\left(S_{n+1}\right)=$ $\operatorname{split}_{n}\left(S_{n}\right)$.

- $D_{n+1} \subseteq\left[s_{n}\right]$ where $[v]=\left\{w \in \omega^{\omega}: w \supseteq v\right\}$.

- $\left|B_{n}\right|=2^{n}$.

Start with $S_{0}=T$ and suppose we have already constructed the tree $S_{n}$. For each $u \in \operatorname{split}_{n}\left(S_{n}\right)$ and $i \in\{0,1\}$ look at the condition $\left(S_{n}\right)_{u^{i}}$. Then there is a condition $U_{n, i} \leq\left(S_{n}\right)_{u^{i}}$ and $t_{u, i} \in \omega^{<\omega}$ such that $U_{n, i} \Vdash t_{n, i} \supseteq s_{n}$ and $\left[t_{n, i}\right] \subseteq \dot{\mathcal{D}}$ (this is possible because $T \Vdash \dot{\mathcal{D}}$ is open dense).

Put then $S_{n+1}=\bigcup\left\{U_{u, i}: u \in \operatorname{split}_{n}\left(S_{n}\right) \wedge i \in\{0,1\}\right\}$ and $D_{n+1}=\left\{t_{n, i}: u \in\right.$ $\left.\operatorname{split}_{n}\left(S_{n}\right) \wedge i \in\{0,1\}\right\}$. Clearly the conditions are satisfied $\left(\left|\operatorname{split}\left(S_{n}\right)\right|=2^{n}\right)$. 
Finally, let $S$ be the fusion of the sequence $\left\langle S_{n}: n \in \omega\right\rangle$ and $\mathcal{D}^{\prime}=\bigcup_{n \in \omega} D_{n}$. Then $S \Vdash \mathcal{D}^{\prime} \subseteq \dot{\mathcal{D}}$ and $\mathcal{D}^{\prime}$ is dense (without loss of generality open dense) in $\omega^{<\omega}$ as we wanted.

Furthermore, we have the following preservation theorem.

Theorem 27 (Shelah [16]) Let $\left\langle\mathbb{P}_{\alpha}, \dot{\mathbb{Q}}_{\alpha}: \alpha<\omega_{2}\right\rangle$ be a countable support iteration

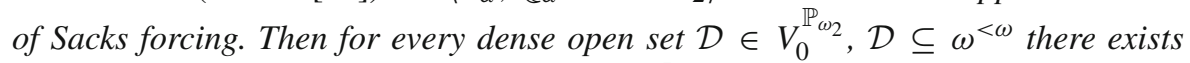
$\mathcal{D}^{\prime} \in V_{0}$ open dense subset of $\omega^{<\omega}$ such that $V^{\mathbb{P}_{\omega_{2}}} \models \mathcal{D}^{\prime} \subseteq \mathcal{D}$.

Recall also the definition of Sacks property for a partial order:

Definition 28 (See Section 6.3.F in [1]) A forcing notion $\mathbb{P}$ has the Sacks Property if for every condition $p \in \mathbb{P}$ and every $\mathbb{P}$-name $\dot{f}$ for an element in $\omega^{\omega}$ there are a condition $q \leq p$ and a slalom $F: \omega \rightarrow[\omega]^{<\omega}$ with $|F(n)| \leq n$ for all $n \in \omega$ such that $q \Vdash \dot{f}(n) \in F(n)$ for all $n \in \omega$.

Note From now on, we will use (without proof) that Sacks forcing has the Sacks property (see Lemma 7.3.2 in [1]) and moreover, that the countable support iteration of proper forcings with the Sacks property also has the Sacks property. For more details of Sacks forcing and the Sacks property see [11].

Proof Of Theorem 27 This uses the fact that a countable support iteration of Sacks forcing has the Sacks property together with the argument we gave for the single step in Lemma 26.

Theorem 29 The generic maximal independent family adjoined by $\mathbb{P}$ over a model of $\mathrm{CH}$ and $2^{\aleph_{0}}=\aleph_{1}$ remains maximal after the countable support iteration of Sacks forcing $\mathbb{S}$ of length $\omega_{2}$.

Proof Let $\left\langle\mathbb{P}_{\alpha}, \dot{\mathbb{Q}}_{\alpha}: \alpha<\omega_{2}\right\rangle$ denote the countable support iteration of $\mathbb{S}$ over $V_{0}=$ $V^{\mathbb{P}}$. We shall prove that the generic maximal independent family $\mathcal{A}$ adjoined by $\mathbb{P}$ is still maximal in the $V^{\mathbb{P} * \mathbb{P}_{\omega_{2}}}$. To do this we will prove the following inductively for all $\alpha<\omega_{2}$ :

$(\star)_{\alpha}$ : In $V^{\mathbb{P} * \mathbb{P}_{\alpha}}$, for all $h \in \mathrm{FF}(\mathcal{A})$ and all $X \subseteq \omega$ such that $X \subseteq \mathcal{A}^{h}$, either $\exists B \in$ $(\operatorname{id}(\mathcal{A}))^{V_{0}}$ such that $\mathcal{A}^{h} \backslash X \subseteq B$, or $\exists h^{\prime} \in \mathrm{FF}(\overline{\mathcal{A}})$ such that $h \subseteq h^{\prime}$ and $\mathcal{A}^{h^{\prime}} \subseteq \mathcal{A}^{h} \backslash X$.

In the following, let $\operatorname{fil}(\mathcal{A})$ denote the dual filter of $\operatorname{id}(\mathcal{A})$. Note that for $h \in \operatorname{FF}(\mathcal{A})$ and $X \subseteq \mathcal{A}^{h}$, there is $B \in \operatorname{id}(\mathcal{A})$ such that $\mathcal{A}^{h} \backslash X \subseteq B$ if and only if there is $F \in \operatorname{fil}(\mathcal{A})$ such that $\mathcal{A}^{h} \cap F \subseteq X$. Note also that $\operatorname{fil}(\mathcal{A})=\left\{F \subseteq \omega: \forall h \in \operatorname{FF}(\mathcal{A}) \exists h^{\prime} \supseteq\right.$ $h$ such that $\mathcal{A}^{h^{\prime}} \subseteq^{*} \bar{F}$ \}.

Now, suppose $(\star)_{\omega_{2}}$ holds. If $\mathcal{A}$ is not maximal in $V^{\mathbb{P} * \mathbb{P}_{\omega_{2}}}$ then there is $X \in V^{\mathbb{P} * \mathbb{P}_{\omega_{2}}} \cap$ $[\omega]^{\omega}$ such that for all $h \in \mathrm{FF}(\mathcal{A})$, both $\mathcal{A}^{h} \cap X$ and $\mathcal{A}^{h} \backslash X$ are infinite. Fix $h$ and consider $Y^{h}=\mathcal{A}^{h} \cap X$. Then $Y^{h} \subseteq \mathcal{A}^{h}$ and so by $(\star)_{\omega_{2}}$ either there is $B \in(\operatorname{id}(\mathcal{A}))^{V_{0}}$ such that $\mathcal{A}^{h} \backslash Y^{h} \subseteq B$, or there is $h^{\prime} \supseteq h$ such that $\mathcal{A}^{h^{\prime}} \subseteq \mathcal{A}^{h} \backslash Y^{h}$. In the latter case, $\mathcal{A}^{h^{\prime}} \cap Y^{h}=\overline{\mathcal{A}}^{h^{\prime}} \cap X=\emptyset$, which is a contradiction to the choice of $X$. In the former case, fix $B \in(\operatorname{id}(\mathcal{A}))^{V_{0}}$ such that $\mathcal{A}^{h} \backslash Y^{h} \subseteq B$. Thus there is $h^{\prime} \in \operatorname{FF}(\mathcal{A})$ such that $h^{\prime} \supseteq h$ and $\mathcal{A}^{h^{\prime}} \cap B=\emptyset$, i.e. $\mathcal{A}^{h^{\prime}} \subseteq \omega \backslash B$. However $\mathcal{A}^{h} \backslash X=\mathcal{A}^{h} \backslash Y^{h} \subseteq B$ and so $\mathcal{A}^{h^{\prime}} \backslash X \subseteq \mathcal{A}^{h} \backslash X \cap \omega \backslash B=\emptyset$, which is again a contradiction to the choice of $X$. 
We are left with the task of proving $(\star)_{\alpha}$ for all $\alpha \leq \omega_{2}$. Proceed inductively.

Base case Fix $h$ and $X$ as in $(\star)_{0}$. If $\mathcal{A}^{h} \backslash X \notin \operatorname{id}(\mathcal{A})$, then there is $h_{0} \in \operatorname{FF}(\mathcal{A})$ such that for all $h_{1} \in \mathrm{FF}(\mathcal{A})$ extending $h_{0}$, the set $\mathcal{A}^{h_{1}} \cap\left(\mathcal{A}^{h} \backslash X\right)$ is infinite. Consider the partition $\mathcal{E}=\left\{\mathcal{A}^{h} \backslash X, \omega \backslash\left(\mathcal{A}^{h} \backslash X\right)\right\}$ and the boolean combination $\mathcal{A}^{h_{0}}$. By Corollary 19.(1) there is $h_{1} \supseteq h_{0}$ such that $\chi\left(\mathcal{E}, \mathcal{A}^{h_{1}}\right)$. However if $\mathcal{A}^{h_{1}} \subseteq \omega \backslash\left(\mathcal{A}^{h} \backslash X\right)$, then $\mathcal{A}^{h_{1}} \cap\left(\mathcal{A}^{h} \backslash X\right)=$ $\emptyset$, which is a contradiction to the choice of $h_{0}$. Thus $\mathcal{A}^{h_{1}} \subseteq \mathcal{A}^{h} \backslash X$, and so $\mathcal{A}^{h_{1}} \cap X=\emptyset$.

Successor case Let $\alpha=\beta+1$. Assume $V^{\mathbb{P} * \mathbb{P}_{\beta}} \vDash(\star)_{\beta}$ and let $G * \bar{G}$ be a $\mathbb{P} * \mathbb{P}_{\beta^{-}}$ generic over $V$ filter. Work in $V_{\beta}=V[G * \bar{G}]$. Suppose there are $p \in \mathbb{Q}_{\beta}\left(=\mathbb{S}_{\beta}\right)$, and a $\mathbb{P}_{\beta}$-name $\dot{X}$ for a subset of $\omega$ that is forced over $V_{\beta}$ by $p$ to be a counterexample for $(\star)_{\alpha}$. Without loss of generality $p$ is also a condition that forces the generic Sacks real added at this step to decide the values of $\dot{X} \cap(n+1)$. Consider the following two cases:

Case 1 Suppose that there is $l \in \omega$ so that, the set $Y_{l}=\left\{m \in \omega: \exists q \leq_{l+1} p\right.$ such that $q \nVdash m \notin \dot{X}$ \} does not belong to the filter fil $(\mathcal{A})$. Since $Y_{l}$ belongs to $V^{\mathbb{P} * \mathbb{P}_{\beta}}$ and $V^{\mathbb{P} * \mathbb{P}_{\beta}} \models Y_{l} \subseteq \mathcal{A}^{h}$ (otherwise, there is $m \in Y_{l} \backslash \mathcal{A}^{h}$ and so, we can find a condition $r \leq p$ for which $r \Vdash m \in \dot{X}$ which is not possible) we can apply the inductive hypothesis $(\star)_{\beta}$ to it. Then we have the following two possibilities:

- Either there is $B_{l} \in \operatorname{id}(\mathcal{A})$ such that $\mathcal{A}^{h} \backslash Y_{l} \subseteq B_{l}$, and now since $Y_{l} \notin \operatorname{fil}(\mathcal{A})$, there is $g \in \mathrm{FF}(\mathcal{A})$ such that for all $g^{\prime} \supseteq g, Y_{l} \backslash \mathcal{A}^{g^{\prime}}$ is infinite. But then we must have that both $h$ and $g$ are compatible, and so we can find $h^{\prime} \in \operatorname{FF}(\mathcal{A})$ a common extension for which we will get $\mathcal{A}^{g} \backslash Y_{l} \subseteq \mathcal{A}^{h}$ is infinite and belongs to $\operatorname{id}(\mathcal{A})$, a contradiction.

- Hence, we must have that there exists $h^{\prime} \supseteq h$ for which $\mathcal{A}^{h^{\prime}} \cap Y_{l}=\emptyset$. This implies that, given $m \in \mathcal{A}^{h^{\prime}}$ for all conditions $q \leq_{l+1} \quad p$ we have $q \Vdash m \notin \dot{X}$ and so, $p \Vdash \dot{X} \cap \mathcal{A}^{h^{\prime}}=\varnothing$. However, this contradicts the assumption that $p$ forces $\dot{X}$ to be a counterexample to $(\star)_{\alpha}$.

Case 2 For all $l \in \omega$ the sets $Y_{l} \in \operatorname{fil}(\mathcal{A})$. Since $\operatorname{fil}(\mathcal{A})$ is a $P$-set, there is $A_{j} \in \operatorname{fil}(\mathcal{A})$ (in fact $A_{j} \in \mathcal{F}_{G}^{0}$ ) such that $A_{j} \subseteq \subseteq^{*} Y_{l}$. Let $f$ be a real in $\omega^{\omega}$ such that $A_{j} \backslash Y_{l} \subseteq f(l)$ for each $l \in \omega$. By the Sacks property, we can assume that $f \in V$.

Refine $A_{j}$ as follows: Take $k_{0}=\min \left(A_{j} \backslash f(0)\right)$ and if we have already chosen $k_{n} \in A_{j}$, let $k_{n+1} \in A_{j}$ such that $k_{n+1}>f(l)$ for all $l \leq n$. Let $B=\left\{k_{n}\right\}_{n \in \omega}$. We can see $B$ as an interval partition $\mathcal{E}$ of $A_{j}$ (which bijectively we can put in correspondence with $\omega$.) Corollary 19. (2) implies that there is $A_{\delta} \in \mathcal{F}_{G}^{0}$ which is a semiselector for $\mathcal{E}$.

Let $\left\{m_{n}\right\}_{n \in \omega}$ be an enumeration of $\mathrm{A}_{\delta}$. It is now enough to show that there is a condition $q \leq p$ such that $q \Vdash A_{\delta} \subseteq \dot{X}$. For this purpose, we construct inductively a fusion sequence $\left\langle q_{n}: n \in \omega\right\rangle \subseteq \mathbb{Q}_{\beta}$ below $p$ such that $q_{n+1} \Vdash m_{n} \in \dot{X}$, for all $n \in \omega$. Then the fusion of such sequence will force that $\dot{X} \in \operatorname{fil}(\mathcal{A})$, contradicting the choice of $p$ as desired.

Start with $q_{0}=p$, and suppose we have constructed $q_{n}$ satisfying the conditions. To complete the inductive construction of the sequence, notice that $m_{n+1} \in Y_{n}\left(m_{n+1}>\right.$ $f(n))$, and so we can choose $q_{n+1} \leq_{n} q_{n}$ such that $q_{n+1} \Vdash m_{n+1} \in \dot{X}$.

Limit case The limit case follows from Theorem [15, Lemma 3.2] of Shelah cited below. For convenience we state the theorem immediately after the current proof, as 
Lemma 30. In addition, we explain why it can be used to complete the preservation of $(\star)_{\alpha}$, for $\alpha$ limit. Note that $\mathcal{F}=\mathcal{F}_{G}^{0} \cup \operatorname{Fr}$ and $\mathcal{H}=\left\{\omega \backslash \mathcal{A}^{h}: h \in \operatorname{FF}(\mathcal{A})\right\}$ satisfy the conditions of Lemma 30 . Note that by Lemma $24, \mathcal{F}$ is Ramsey.

To see (2) consider any $Z \in \mathcal{P}(\omega) \backslash \operatorname{fil}(\mathcal{A})$. Thus $\omega \backslash Z \notin \operatorname{id}(\mathcal{A})$ and so there is $h \in \operatorname{FF}(\mathcal{A})$ such that for al $h^{\prime} \supseteq h,\left|\mathcal{A}^{h^{\prime}} \cap(\omega \backslash Z)\right|=\left|\mathcal{A}^{h^{\prime}} \backslash Z\right|=\omega$. Consider the set $Y=\mathcal{A}^{h} \backslash Z$. Thus $Y \subseteq \mathcal{A}^{\bar{h}}$ and so by $(\star)_{\alpha}$ either $\exists B \in(\operatorname{id}(\mathcal{A}))^{V_{0}}$ such that $\mathcal{A}^{h} \backslash Y \subseteq B$ and so $\mathcal{A}^{h} \backslash Y \in \operatorname{id}(\mathcal{A})$, or $\exists h^{\prime} \supseteq h$ such that $\mathcal{A}^{h^{\prime}} \subseteq \mathcal{A}^{h} \backslash Y$. In the latter case, $\mathcal{A}^{h^{\prime}} \subseteq \mathcal{A}^{h} \backslash Y=Z \cap \mathcal{A}^{h}$ and so $\mathcal{A}^{h^{\prime}} \backslash Z=\emptyset$, which is a contradiction. Therefore $\mathcal{A}^{h} \backslash Y \in \operatorname{id}(\mathcal{A})$ and so there is $h^{\prime} \supseteq h$ such that $\mathcal{A}^{h^{\prime}} \cap \mathcal{A}^{h} \cap Z=\mathcal{A}^{h^{\prime}} \cap Z$ is finite and so $Z \subseteq \subseteq^{*} \omega \backslash \mathcal{A}^{h^{\prime}}$. Take $X=\omega \backslash \mathcal{A}^{h^{\prime}}$.

Lemma 30 (Shelah, Lemma 3.2 in [15]). Let $\mathcal{F}$ and $\mathcal{H}$ be families of subsets of $\omega$ such that:

(1) $\mathcal{F}$ contains all co-infinite sets, every element $\mathcal{F}$ is non-empty, $\mathcal{F}$ is closed under finite intersections, every countable $\mathcal{G} \subseteq \mathcal{F}$ has a pseudointersection in $\mathcal{F}$ and $\mathcal{F}$ is Ramsey.

(2) $\mathcal{H} \subseteq \mathcal{P}(\omega) \backslash\langle\mathcal{F}\rangle$, where $\langle\mathcal{F}\rangle$ is the filter generated by $\mathcal{F}$ and

$$
\mathcal{P}(\omega) \backslash\langle\mathcal{F}\rangle \subseteq\left\{Z \subseteq \omega: \text { there exists } X \in \mathcal{H} \text { with } Z \subseteq^{*} X\right\}
$$

If $\left\langle\mathbb{P}_{\alpha}, \dot{\mathbb{Q}}_{\alpha}: \alpha<\delta\right\rangle$ is a countable support iteration of $\omega^{\omega}$-bounding proper posets such that for all $\alpha<\delta$,

$$
\Vdash_{\mathbb{P}_{\alpha}}(\mathcal{P}(\omega) \backslash\langle\mathcal{F}\rangle) \subseteq\left\{Z \subseteq \omega: \exists X \in \mathcal{H} \text { such that } Z \subseteq \subseteq^{*} X\right\}
$$

then the same holds for $\alpha=\delta$.

The above proof clearly shows that the generic maximal independent family adjoined by $\mathbb{P}$ over a model of GCH remains also maximal after the countable support product of Sacks forcing.

\section{Ideals and independence}

Throughout the section, we study the relationship between the independence diagonalization ideal and the independence density ideals. For convenience, we restate their definitions. Given an independent family $\mathcal{A}$, an ideal $\mathcal{J}_{\mathcal{A}} \subseteq[\omega]^{\leq \omega}$ is said to be a diagonalization ideal, if

(1) $\mathcal{J}_{\mathcal{A}} \cap\left\{\mathcal{A}^{h}: h \in \mathrm{FF}(\mathcal{A})\right\}=\emptyset$.

(2) For every $X \in[\omega]^{\omega} \cap V$ there is $h \in \mathrm{FF}(\mathcal{A})$ such that $X \cap \mathcal{A}^{h}$ or $\mathcal{A}^{h} \backslash X$ belongs to $\mathcal{J}_{\mathcal{A}}$.

On the other hand, given an independent family $\mathcal{A}$, we defined the independence density ideal, $\operatorname{id}(\mathcal{A})$, as the set of all $X \subseteq \omega$ such that $\forall h \in \operatorname{FF}(\mathcal{A}) \exists h^{\prime} \supseteq h$ such that $\mathcal{A}^{h^{\prime}} \cap X$ is finite.

Lemma 31 Let $\mathcal{A}$ be an independent family. Then $\operatorname{id}(\mathcal{A}) \subseteq \mathcal{J}_{\mathcal{A}}$. 
Proof Let $\left\langle\mathcal{J}_{\alpha}: \alpha<\mathfrak{c}\right\rangle$ be an increasing sequence of ideals associated to a fixed enumeration $\left\{X_{\alpha}\right\}_{\alpha \in \mathfrak{c}}$ of $\mathcal{P}(\omega)$ and $\mathcal{A}$ as in Lemma 3, such that $\mathcal{J}_{\mathcal{A}}=\bigcup_{\alpha<\mathfrak{c}} \mathcal{J}_{\alpha}$. Suppose towards a contradiction that there is $X \in \operatorname{id}(\mathcal{A})$ and $X \notin \mathcal{J}_{\mathcal{A}}$. Clearly, $X \notin[\omega]^{<\omega}$. Then for some $\alpha, X_{\alpha}=X$ and since $X \notin \mathcal{J}_{\alpha+1}$, there must be $h_{0} \in \operatorname{FF}(\mathcal{A})$ and $Y \in \mathcal{J}_{\alpha}$ such that $\mathcal{A}^{h} \subseteq X \cup Y$. Therefore $\mathcal{A}^{h} \backslash X \subseteq Y$ and so $\mathcal{A}^{h} \backslash X \in \mathcal{J}_{\alpha} \subseteq \mathcal{J}_{\mathcal{A}}$. By hypothesis, $X \in \operatorname{id}(\mathcal{A})$ and so there is $h^{\prime} \supseteq h$ such that $\mathcal{A}^{h^{\prime}} \cap X$ is finite. However $\mathcal{A}^{h^{\prime}} \subseteq \mathcal{A}^{h}$ and so $\mathcal{A}^{h^{\prime}} \backslash X \in \mathcal{J}_{\mathcal{A}}$. But then $\mathcal{A}^{h^{\prime}}=\left(\mathcal{A}^{h^{\prime}} \backslash X\right) \cup\left(\mathcal{A}^{h^{\prime}} \cap X\right) \in \mathcal{J}_{\mathcal{A}}$, which is a contradiction.

Lemma 32 If $\mathcal{A}$ is an independent family which is not maximal, then $\operatorname{id}(\mathcal{A}) \subsetneq \mathcal{J}_{\mathcal{A}}$.

Proof Fix $X \in[\omega]^{\omega} \backslash \mathcal{A}$ such that $\{X\} \cup \mathcal{A}$ is independent. By definition of $\mathcal{J}_{\mathcal{A}}$, there is $h \in \operatorname{FF}(\mathcal{A})$ such that $X \cap \mathcal{A}^{h}$ of $\mathcal{A}^{h} \backslash X$ is in $\mathcal{J}_{\mathcal{A}}$. Since neither of them is $\operatorname{in} \operatorname{id}(\mathcal{A})$, we obtain the desired claim.

The above Lemma implies in particular that the density ideal is not necessarily a diagonalization ideal. However, for the following class of maximal independent families, the density and diagonalization ideals coincide.

Definition 33 An independent family $\mathcal{A}$ is said to be densely maximal if for every $X \in[\omega]^{\omega} \backslash \mathcal{A}$ and every $h \in \operatorname{FF}(\mathcal{A})$, there is $h^{\prime} \in \mathrm{FF}(\mathcal{A})$ for which either $X \cap \mathcal{A}^{h^{\prime}}$ of $\mathcal{A}^{h^{\prime}} \backslash X$ is finite.

Lemma 34 An infinite independent family $\mathcal{A}$ is densely maximal if and only if the following property holds:

$(\star)$ For all $h \in \operatorname{FF}(\mathcal{A})$ and all $X \subseteq \omega$ such that $X \subseteq \mathcal{A}^{h}$, either $\exists B \in \operatorname{id}(\mathcal{A})$ such that $\mathcal{A}^{h} \backslash X \subseteq B$, or $\exists h^{\prime} \in \mathrm{FF}(\mathcal{A})$ such that $h \subseteq h^{\prime}$ and $\mathcal{A}^{h^{\prime}} \subseteq \mathcal{A}^{h} \backslash X$.

Proof Suppose $\mathcal{A}$ satisfies property $(\star)$. Let $X \in[\omega]^{\omega}, h \in \mathrm{FF}(\mathcal{A})$ and consider $Y=X \cap \mathcal{A}^{h}$. Then by $(\star)$ either there is $B \in \operatorname{id}(\mathcal{A})$ such that $\mathcal{A}^{h} \backslash Y=\mathcal{A}^{h} \backslash X \subseteq$ $B$, in which case $\mathcal{A}^{h} \backslash Y$ belongs to $\operatorname{id}(\mathcal{A})$ itself and so there is $h^{\prime} \supseteq h$ such that $\mathcal{A}^{h^{\prime}} \cap\left(\mathcal{A}^{h} \backslash X\right)=\mathcal{A}^{h^{\prime}} \backslash X$ is finite, or there is $h^{\prime \prime} \supseteq h$ such that $\mathcal{A}^{h^{\prime \prime}} \subseteq \mathcal{A}^{\bar{h}} \backslash Y=\mathcal{A}^{h} \backslash X$, which implies $\mathcal{A}^{h^{\prime \prime}} \cap X=\emptyset$. Thus, $\mathcal{A}$ is densely maximal.

Now, suppose $\mathcal{A}$ is densely maximal. Fix $h \in \mathrm{FF}(\mathcal{A})$ and $X \subseteq \mathcal{A}^{h}$. We will show that $\mathcal{A}$ satisfies property $(\star)$. Suppose, there is no $B \in \operatorname{id}(\mathcal{A})$ such that $\mathcal{A}^{h} \backslash X \subseteq B$. Thus in particular $\mathcal{A}^{h} \backslash X \notin \operatorname{id}(\mathcal{A})$ and so there is $h^{\prime} \in \operatorname{FF}(\mathcal{A})$ such that for all $h^{\prime \prime} \supseteq h^{\prime}$, the set $\mathcal{A}^{h^{\prime \prime}} \cap\left(\mathcal{A}^{h} \backslash X\right)$ is infinite. If $h^{\prime}$ and $h$ are incompatible (as conditions in $\operatorname{FF}(\mathcal{A})$ ), then $\mathcal{A}^{h^{\prime}} \cap\left(\mathcal{A}^{h} \backslash X\right)=\emptyset$, which is a contradiction. Therefore $h^{\prime}$ and $h$ are compatible. Without loss of generality $h^{\prime} \supseteq h$ and so we have that there is $h^{\prime} \supseteq h$ such that for all $h^{\prime \prime} \supseteq h^{\prime},\left|\mathcal{A}^{h^{\prime \prime}} \backslash X\right|=\omega$. Apply the property of $\mathcal{B}$ being densely maximal to $\mathcal{A}^{h^{\prime}} \backslash X$ and $h^{\prime}$. Thus there is $h^{\prime \prime} \supseteq h^{\prime}$ such that either $\mathcal{A}^{h^{\prime \prime}} \cap\left(\mathcal{A}^{h^{\prime}} \backslash X\right)=\mathcal{A}^{h^{\prime \prime}} \backslash X$ or $\mathcal{A}^{h^{\prime \prime}} \backslash\left(\mathcal{A}^{h^{\prime}} \backslash X\right)=\mathcal{A}^{h^{\prime \prime}} \cap X$ is finite. Therefore, the latter must hold. But then, there is $h^{\prime \prime \prime} \supseteq h^{\prime \prime}$ such that $\mathcal{A}^{h^{\prime \prime \prime}} \cap X=\emptyset$. That is, there is $h^{\prime \prime \prime} \supseteq h$ such that $\mathcal{A}^{h^{\prime \prime \prime}} \subseteq \mathcal{A}^{h} \backslash X$ and so $(\star)$ holds. 
Lemma 35 Let $\mathcal{A}$ be a densely maximal independent family and let $\mathcal{J}$ be an ideal on $\omega$ such that $\mathcal{J} \cap\left\{\mathcal{A}^{h}: h \in \operatorname{FF}(\mathcal{A})\right\}=\emptyset$. Then $\mathcal{J} \subseteq \operatorname{id}(\mathcal{A})$.

Proof Let $X \in \mathcal{J}$. Suppose $X \notin \operatorname{id}(\mathcal{A})$. Then there is $g \in \operatorname{FF}(\mathcal{A})$ such that for all $g^{\prime} \supseteq g$, the set $\mathcal{A}^{g^{\prime}} \cap X$ is infinite. Applying dense maximality to $X$ and $g$, we can find $g^{\prime} \supseteq g$ such that $X \cap \mathcal{A}^{g^{\prime}}$ or $\mathcal{A}^{g^{\prime}} \backslash X$ is finite. Thus $\mathcal{A}^{g^{\prime}} \backslash X$ is finite. Since $\mathcal{A}$ is infinite, there is $g^{\prime \prime} \supseteq g^{\prime}$ such that $\mathcal{A}^{g^{\prime \prime}} \cap(\omega \backslash X)=\emptyset$, i.e. $\mathcal{A}^{g^{\prime \prime}} \subseteq X$. Thus $\mathcal{A}^{g^{\prime \prime}} \in \mathcal{J}$, which is a contradiction.

Corollary 36 If $\mathcal{A}$ is densely maximal independent, then $\mathcal{J}_{\mathcal{A}} \subseteq \operatorname{id}(\mathcal{A})$ and so $\mathcal{J}_{\mathcal{A}}=$ $\operatorname{id}(\mathcal{A})$.

As a corollary to Shelah's [15, Lemma 3.2], we obtain:

Corollary 37 A densely maximal independent family $\mathcal{A}$ such that the dual filter of its diagonalization ideal $\operatorname{id}(\mathcal{A})$ is generated by a Ramsey filter and the co-finite sets remains maximal after the countable support iteration of Sacks forcing, as well as after the countable support product of Sacks forcing.

Finally, we prove that neither the density, nor the diagonalization ideal is maximal.

Proposition 38 Given an independent family $\mathcal{A}$ the ideal $\mathcal{J}_{\mathcal{A}}$ is not maximal in the following sense: There is a set $X \in[\omega]^{\omega} \backslash\left\{\mathcal{A}^{h}: h \in \mathrm{FF}(\mathcal{A})\right\}$ such that neither $X$ nor $\omega \backslash X$ belongs to $\mathcal{J}_{\mathcal{A}}$.

Proof Let $g \in \mathrm{FF}(\mathcal{A})$, let $x_{0}$ be a finite, non-empty set disjoint from $\mathcal{A}^{g}$ and let $X=\mathcal{A}^{g} \cup x_{0}$. Given $h \in \operatorname{FF}(\mathcal{A})$, denote by $h^{\perp}$ the element in $\operatorname{FF}(\mathcal{A})$ with same domain as $h$, and such that for all $B \in \operatorname{dom}(h)$, if $h(B)=B$ then $h^{\perp}(B)=\omega \backslash B$, and if $h(B)=\omega \backslash B$, then $h^{\perp}(B)=B$.

Note that $X \notin\left\{\mathcal{A}^{h}: h \in \mathrm{FF}(\mathcal{A})\right\}$. Indeed, suppose there is $h \in \mathrm{FF}(\mathcal{A})$ for which $X=\mathcal{A}^{g} \cup x_{0}=\mathcal{B}^{h}$. Then $h \subseteq g$ and $h \neq g$. Let $w=\operatorname{dom}(g) \backslash \operatorname{dom}(h)$ and define $h^{\prime}=h \cup\left(g\lceil w)^{\perp}\right.$. But then, $\mathcal{A}^{h^{\prime}}=\mathcal{A}^{h} \cap \mathcal{A}^{\left(g\lceil w)^{\perp}\right.}=\left(\mathcal{A}^{g} \cup x_{0}\right) \cap \mathcal{A}^{\left(g\lceil w)^{\perp}\right.}=$ $\mathcal{A}^{\left(g\lceil w)^{\perp}\right.} \cap x_{0}$ must be infinite, which is a contradiction. In addition, since $\mathcal{J}_{\mathcal{A}}$ is an ideal and $\mathcal{A}^{g} \notin \mathcal{J}_{\mathcal{A}}, X \notin \mathcal{J}_{\mathcal{A}}$. Finally note that $\mathcal{A}^{g^{\perp}} \subseteq \subseteq^{*} \omega \backslash X$ and since $\mathcal{A}^{g^{\perp}} \notin \mathcal{J}_{\mathcal{A}}$, the set $\omega \backslash X$ does not belong to $\mathcal{J}_{\mathcal{A}}$.

\section{Final remarks}

Shelah's proof of $\mathfrak{i}<\mathfrak{u}$ from [15] gives the existence, under $\mathrm{CH}$, of a decreasing sequence of conditions $\left\{\left(\mathcal{A}_{\alpha}, A_{\alpha}\right)\right\}_{\alpha \in \omega_{1}}$ in the countably closed poset $\mathbb{P}$ from Sect. 3, with the property that

- $\mathcal{A}_{\omega_{2}}=\bigcup_{\alpha \in \omega_{1}} \mathcal{A}_{\alpha}$ satisfies property $(\star)$ and so in our terminology is densely maximal and,

- the filter generated by the tower $\tau=\left\{A_{\alpha}\right\}_{\alpha \in \omega}$ is Ramsey. 
Furthermore, $\tau$ is the dual filter to $\operatorname{id}\left(\mathcal{A}_{\omega_{2}}\right){ }^{4}$ Thus, in particular, $\mathrm{CH}$ implies the existence of a Sacks indestructible maximal independent family. This approach will be used in a forthcoming paper to show the existence of a co-analytic Sacks indestructible maximal independent family and so the consistency of the existence of a co-analytic maximal independent family with arbitrarily large continuum. Another immediate consequence of Corollary 36 and Lemma 9 that is that $\mathrm{CH}$ implies the existence of a maximal independent family for which the diagonalization partial order does adjoin a dominating real.

We conclude with some open questions, which naturally follow from the presented theory.

Question 1: If we define $\mathfrak{i}_{d}$ to be the minimal size of a densely maximal independent family, clearly $\mathfrak{i} \leq \mathfrak{i}_{d}$. However of interest remains the following: Is it consistent that $\mathfrak{i}<\mathfrak{i}_{d}$ ?

Question 2: Both of the constructions of densely maximal independent families, which we discussed in the paper relay on $\mathrm{CH}$. Thus one may ask: Is is consistent that there are no densely maximal independent families?

Question 3: Is there a Sacks indestructible independent family which is not densely maximal?

Acknowledgements Open access funding provided by Austrian Science Fund (FWF).

Open Access This article is distributed under the terms of the Creative Commons Attribution 4.0 International License (http://creativecommons.org/licenses/by/4.0/), which permits unrestricted use, distribution, and reproduction in any medium, provided you give appropriate credit to the original author(s) and the source, provide a link to the Creative Commons license, and indicate if changes were made.

\section{References}

1. Bartoszyński, T., Judah, H.: Set Theory. On the Structure of the Real Line, p. xii+546. A K Peters Ltd., Wellesley (1995)

2. Blass, A.: Combinatorial Characteristics of the Continuum. Handbook of Set Theory, vol. 1,2,3, pp. 395-489. Springer, Dordrecht (2010)

3. Brendle, J., Fischer, V.: Mad families, splitting families and large continuum. J. Symb. Log. 76(1), 198-208 (2011)

4. Brendle, J., Fischer, V., Khomskii, Y.: Definable maximal independent families. Trans. Am. Math. Soc. https://doi.org/10.1090/proc/14497

5. Ciesielski, K., Pawlikowski, J.: The Covering Property Axiom, CPA. Cambridge Tracks in Mathematics, vol. 164. Cambridge University Press, Cambridge (2004)

6. Fichtenholz, G., Kantorovitch, L.: Sur les opérations linéaires dans l'espace des fonctions bornées. Stud. Math. 5, 69-98 (1935)

7. Fischer, V., Shelah, S.: The spectrum of independence. Arch. Math. Logic. https://doi.org/10.1007/ s00153-019-00665-y

8. Fischer, V., Schilhan, J.: Definable towers, (submitted)

9. Fischer, V., Törnquist, A.: Template iterations and maximal cofinitary groups. Fundam. Math. 230(3), 205-236 (2015)

10. Geschke, S.: Almost disjoint and independent families. RIMS Kokyuroku 1790, 1-9 (2012)

\footnotetext{
4 One of the differences between the $\mathrm{CH}$ inductive construction and the generic Sacks indestructible maximal independent family is that the dual filter of the density ideal of the latter is not necessarily generated by a tower.
} 
11. Geschke, S., Quickert, S.: On Sacks forcing and the Sacks property. In: Foundations of the Formal Sciences III, Complexity in Mathematics and Computer Science, Papers of a Conference in Vienna, September 21-24, 2001. Kluwer Academic Publishers, Dordrecht (2004)

12. Halbeisen, L.: Combinatorial Set Theory. With a Gentle Introduction to Forcing. Springer Monographs in Mathematics, p. xvi+453. Springer, London (2012)

13. Hausdorff, F.: Über zwei Sätze von G. Fichtenholz und L. Kantorovitch. Stud. Math. 6, 18-19 (1936)

14. Kunen, K.: Set theory. Studies in Logic (London), vol. 34, p. viii+401. College Publications, London (2011)

15. Shelah, S.: Con $(\mathfrak{u}>$ i). Arch. Math. Log. 31(6), 433-443 (1992)

16. Shelah, S.: Proper and Improper Forcing, Perspectives in Mathematical Logic, second edn. Springer, Berlin (1998)

Publisher's Note Springer Nature remains neutral with regard to jurisdictional claims in published maps and institutional affiliations. 